\begin{tabular}{|c|l|}
\hline Title & $\begin{array}{l}\text { Interference effects in the sum frequency generation spectra of thin organic films. I. Theoretical modeling and } \\
\text { simulation }\end{array}$ \\
\hline Author(s) & Tong, Yujin; Zhao, Y anbao; Li, Na; Osawa, Masatoshi; Davies, Paul B.; Y e, Shen \\
\hline Citation & $\begin{array}{l}\text { Journal of Chemical Physics, 133(3), 034704 } \\
\text { https://doi.org/10.1063/13428668 }\end{array}$ \\
\hline Issue Date & 2010-07-21 \\
\hline Doc URL & http://hdl.handle.net/2115/43793 \\
\hline Rights & $\begin{array}{l}\text { Copyright 2010 A merican Institute of Physics. This article may be downloaded for personal use only. A ny other use } \\
\text { requires prior permission of the author and the A merican Institute of Physics. The following article appeared in J. } \\
\text { Chem. Phys. 133, 034704 (2010) and may be found at https:/dx.doi.org/10.1063/.3428668 }\end{array}$ \\
\hline Type & article \\
\hline File Information & JCP133 3_034704.pdf \\
\hline
\end{tabular}

Instructions for use 


\title{
Interference effects in the sum frequency generation spectra of thin organic films. I. Theoretical modeling and simulation
}

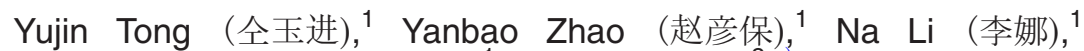 \\ Masatoshi Osawa (大澤雅俊), ${ }^{1}$ Paul B. Davies, ${ }^{2, a)}$ and Shen Ye (叶深) ${ }^{1,3, a)}$ \\ ${ }^{1}$ Catalysis Research Center, Hokkaido University, Sapporo 001-0021, Japan \\ ${ }^{2}$ Department of Chemistry, University of Cambridge, Lensfield Road, Cambridge CB2 IEW, \\ United Kingdom \\ ${ }^{3}$ PRESTO, Japan Science and Technology Agency (JST), Tokyo Japan
}

(Received 28 January 2010; accepted 9 March 2010; published online 16 July 2010)

\begin{abstract}
A general theoretical calculation is described for predicting the interference effect in the sum frequency generation (SFG) spectra from a model thin-film system as a function of film thickness. The calculations were carried out for a three-layer thin film consisting of an organic monolayer, a dielectric thin film of variable thickness, and a gold substrate. This system comprises two sources of SFG, namely, a resonant contribution from the monolayer/dielectric film interface and a nonresonant contribution from the dielectric film/gold interface. The calculation shows that both the spectral intensity and the shape of the SFG spectra vary significantly with the thickness of the dielectric layer due to interference effects in the thin film. The intensity changes at a particular frequency were explained in terms of the changes in the local field factors ( $L$ factors) as a function of the dielectric film thickness. The $L$ factor for each beam changes periodically with the thickness of the dielectric film. However, the combined $L$ factor for the three beams shows complicated thickness dependent features and no clear periodicity was found. On the other hand, if the susceptibilities of both the resonant and nonresonant terms are fixed, changes in the spectral shape will be mainly due to changes in the phase differences between the two terms with the film thickness. The interference behavior also depends strongly on the polarization combinations of the sum frequency, visible, and infrared beams. A general method is provided for predicting changes in the spectral shapes at different film thicknesses by taking into account the relative intensities and phases of the SFG signals from the two interfaces. The model calculation provides important insights for understanding the nonlinear optical responses from any thin-film system and is an essential tool for quantitatively revealing the nonlinear susceptibilities, which are directly related to the actual structure of the interfacial molecules from the observed SFG spectra after quantitative removal of the $L$ factors. (C) 2010 American Institute of Physics. [doi:10.1063/1.3428668]
\end{abstract}

\section{INTRODUCTION}

The second-order nonlinear optical technique of sum frequency generation (SFG) vibrational spectroscopy has become a powerful tool in surface science. ${ }^{1-4}$ SFG possesses almost all the advantages that conventional infrared (IR) and Raman vibrational spectroscopy have, while in addition exhibiting surface/interface specificity, submonolayer sensitivity, and time resolution. In the past 2 decades, SFG has been widely employed to investigate molecular structures at various surfaces and interfaces including self-assembled monolayers (SAMs) ${ }^{5-12}$ thin polymer films, ${ }^{13-15}$ and LangmuirBlodgett (LB) films. ${ }^{16-20}$ Since SFG is a coherent optical process, interference effects are inherently involved in the SFG observation. These include the interference between the resonant and nonresonant SFG signals, ${ }^{21-24}$ interference between different vibrational modes, ${ }^{25-27}$ and interference between SFG signals from different polarization combinations. ${ }^{28,29}$ In particular, when the film thickness is comparable to the wavelength of the pumped or emitted light

${ }^{\text {a)} E l e c t r o n i c ~ a d d r e s s e s: ~ p b d 2 @ c a m . a c . u k ~ a n d ~ y e @ c a t . h o k u d a i . a c . j p . ~}$ beams, the thickness interference of the light beams due to the thin film will significantly affect the observed SFG spectra. ${ }^{15,30-33}$ Without quantitative analysis of the interference effects, it is not possible to obtain quantitative structural information about the film from the SFG spectra.

Many studies of thickness interference effects in surface optical techniques such as SFG and second harmonic generation (SHG) have been reported. ${ }^{34-38}$ Feller et al. gave a comprehensive derivation of how film thickness interference affects the SHG signals from a liquid-crystal/polymer/ $/ \mathrm{SiO}_{2}$ thin-film system by considering multiple reflections within the thin polymer film. They were able to acquire accurate molecular orientational information on the polymer surface based on their model. ${ }^{39}$ Wilson et al. ${ }^{40,41}$ characterized the thickness interference effect on the SFG signals from a threelayer system comprising polymer/glass/metal, and demonstrated that by controlling the film's thickness they could selectively probe the molecular structure at the free polymer/ air or buried polymer/glass interface. Clark ${ }^{33}$ studied the thickness effect from a thin polymer film on a dielectric substrate surface and pointed out that all the $\mathrm{C}-\mathrm{H}$ stretching modes showed similar interference dependences, which al- 
lowed them to select an optimal thickness to obtain the strongest SFG signal. Ye et al. ${ }^{15}$ also demonstrated that molecular structure from different interfaces of a polymer $_{1} /$ polymer $_{2} /$ metal model system can be selectively determined by controlling the thickness of one of the polymers in the system.

Alternatively, by constructing appropriate model systems and assuming that the molecules on the surface or at the interface have a particular orientation and conformation, one can directly simulate SFG spectra at specific film thicknesses. Hirose et al. proposed a method to simulate the SFG intensity of $\mathrm{CH}$ stretching modes from a multilayer of formic acid growing on a $\operatorname{Pt}(110)-(1 \times 2)$ surface under ultrahigh vacuum. Their calculation showed good agreement with the experimentally observed thickness dependence of the SFG signals at a fixed frequency. ${ }^{32}$ With this method, the authors demonstrated that the growth rate of the formic acid thin film on the surface can be quantitatively determined from SFG observations. ${ }^{42}$ Later, Lambert et al. $^{31}$ developed a model to simulate the interference effect in the SFG spectra from a monolayer/dielectric thin-film/gold substrate system. They showed that the SFG spectra of the $\mathrm{CH}$ stretching modes of the methyl $\left(\mathrm{CH}_{3}\right)$ groups in an octadecylsiloxane monolayer on the dielectric thin-film surface changed substantially with the thickness of the film. Their calculation revealed that the phase of the antisymmetric $\left(\mathrm{r}^{-}\right)$stretching mode of the $\mathrm{CH}_{3}$ group became inverted with a thickness periodicity of 162 $\mathrm{nm}$ as the thickness of the dielectric film changes. This was much shorter than that for the symmetric $\left(\mathrm{r}^{+}\right)$mode periodicity of $3.01 \mu \mathrm{m} .{ }^{43,44}$ As mentioned later by the same authors, the possibility of multiple reflections within the thin film, which can degrade the quality of the simulated spectra, was not included in the calculation. Further improvement of the model by taking into account multiple reflections in the dielectric layer was reported later. ${ }^{45}$ However, since the calculation and experiments were carried out using a counterpropagating beam geometry, these results are difficult to compare directly with most of the recent SFG results, which have been obtained with a copropagating beam geometry.

As mentioned above, although the general theory for the interference in the SFG signals arising from a thin-layer system has already been established, there is still a gap between theory and experiment due to the complicated numerical calculation procedure required. Furthermore, it is difficult to compare the calculated results from different workers since most of the calculation programs are not publicly available. This situation impedes further application of the SFG technique in several fields. It is clearly desirable to generate a common program to quantitatively evaluate the effect of thickness related interference on the SFG spectra under a variety of conditions for different thin-film systems.

In the present study we have developed a general model calculation for the effect of thickness generated interference on SFG spectra for a single thin-film system based on models already reported in the literature. ${ }^{31,39}$ After a brief introduction to the theory of SFG for a thin-layer system, we calculate the SFG spectra for the $\mathrm{C}-\mathrm{H}$ stretching modes of the terminal $\mathrm{CH}_{3}$ groups of a monolayer of fatty acid molecules with a long alkyl chain, deposited on top of a thin dielectric layer of variable thickness on a gold substrate. This calculation is performed as a function of the thickness of the dielectric layer for different polarization combinations under a copropagating incident beam geometry. This paper focuses on the modeling, calculation, and resulting theoretical spectral features, while a detailed comparison of the simulated and actual experimental spectra for different thin-film systems are presented in a subsequent paper. ${ }^{46}$ The source codes for the model calculation are available from the authors on request. We anticipate that this will aid the understanding and further application of SFG spectroscopy to thin-layer system.

\section{THEORY: GENERAL BACKGROUND FOR SFG FROM A THIN LAYER SYSTEM}

The basic theory of the thickness interference effect in SFG spectroscopy has already been established. ${ }^{36-38}$ In order to simulate thickness dependent SFG spectra, it is first necessary to construct a theoretical model that resembles a real thin-film system. One can then simulate the thickness dependent Fresnel coefficients of each of the electric fields at the interface and the frequency dependent second-order nonlinear susceptibilities of the SF active molecules. Combining these produces the SFG spectra at different thicknesses. Although the general theory on thickness dependent interference has been discussed previously, for completeness a systematic framework of how the SFG spectral features are simulated will be described here.

Figure 1(a) shows a schematic of the sum frequency generated by a single interface between media 1 and 2 . The SFG intensity is proportional to the intensities of the incident visible and IR beams and to the square of the second-order nonlinear susceptibilities $\left(\chi_{i j k}^{(2)}\right)$ of the interface, ${ }^{34}$

$$
I_{\mathrm{SFG}}\left(\omega_{s}\right) \propto\left|\sum_{i} \sum_{j, k} L_{i i}\left(\omega_{\mathrm{SFG}}\right) \chi_{i j k}^{(2)} L_{j j}\left(\omega_{\mathrm{vis}}\right) L_{k k}\left(\omega_{\mathrm{IR}}\right)\right|^{2} I_{\mathrm{vis}} I_{\mathrm{IR}},
$$

where $(i, j, k=x, y$, or $z)$ are the coordinates in the interfacefixed reference frame; $I_{\mathrm{SFG}}, I_{\mathrm{vis}}$, and $I_{\mathrm{IR}}$ are the power densities of the SFG, visible, and IR beams, respectively; $\chi_{i j k}^{(2)}$ is the second-order susceptibility of the interface and comprises a third rank tensor containing 27 elements. For an azimuthally isotropic interface, only four independent nonvanishing components $\chi_{z z z}^{(2)}, \chi_{x x z}^{(2)}\left(=\chi_{y y z}^{(2)}\right), \chi_{x z x}^{(2)}\left(=\chi_{y z y}^{(2)}\right)$, and $\chi_{z x x}^{(2)}\left(=\chi_{z y y}^{(2)}\right)$ are left. $^{47,48}$ These $\chi_{i j k}^{(2)}$ s normally contain two terms, namely, a nonresonant part, $\chi_{\mathrm{NR}}^{(2)}$, and a resonant part, $\chi_{R S, q^{*}}^{(2)} \chi_{\mathrm{NR}}^{(2)}$ arises from higher order contributions such as the electric quadrupole moment and magnetic dipole moment of matter and usually shows little dependence on the IR frequencies but is dependent on the visible frequency. ${ }^{3,4} \chi_{\mathrm{NR}}^{(2)}$ is negligible for many dielectric bulk materials with inversion symmetry such as glass, fused quartz, and calcium fluoride $\left(\mathrm{CaF}_{2}\right)$ but is reasonably large for some metal substrates such as gold and silver. On the other hand, $\chi_{R S, q}^{(2)}$ carries surface structural information such as molecular density, orientation, and conformation on the surface and can be related to the microscopic (molecular) properties of the molecules via $^{34}$ 
(a)

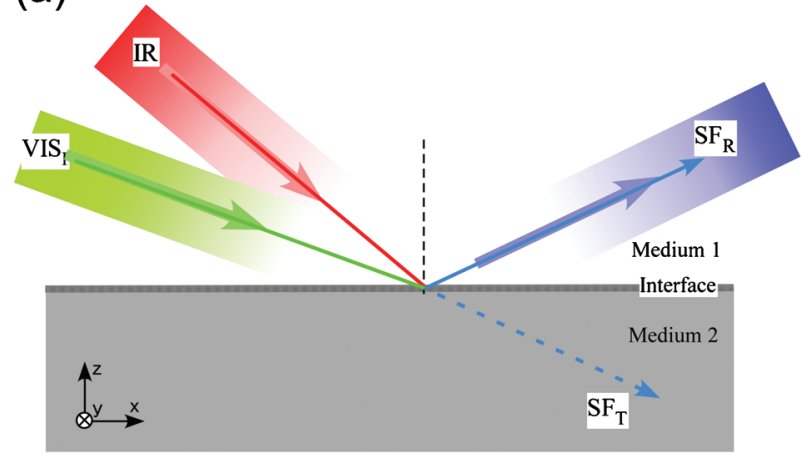

(b)

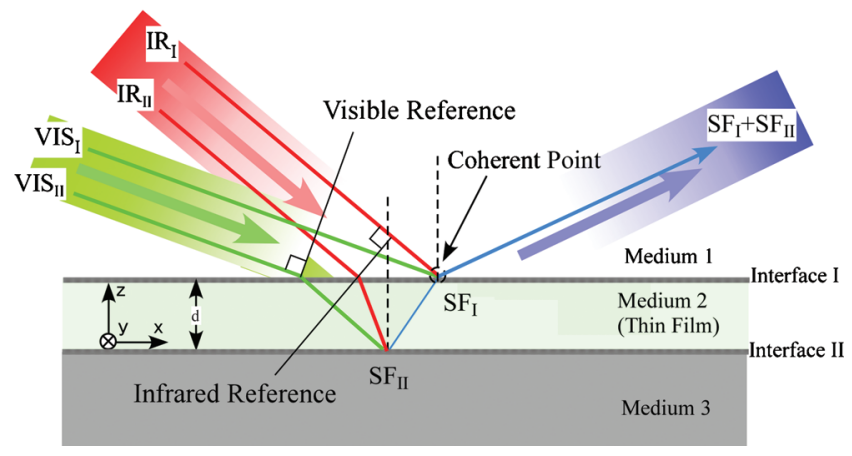

FIG. 1. (a) Schematic for SFG from an interface between media 1 and 2. (b) A general interference model for SFG from a thin-film system on a solid substrate (medium 3) consisting of two interfaces. For simplification, multiply reflected beams are not shown in the figure.

$$
\chi_{i j k, q}^{(2)}=N_{s} \sum_{\xi, \eta, \zeta}\langle(\hat{i} \cdot \hat{\xi})(\hat{j} \cdot \hat{\eta})(\hat{k} \cdot \hat{\zeta})\rangle \beta_{\xi \eta \zeta}^{(2)}
$$

where $N_{s}$ is the number of molecules at the interface. $(\hat{i} \cdot \hat{\xi})(\hat{j} \cdot \hat{\eta})(\hat{k} \cdot \hat{\zeta})$ are the Euler transformation matrices between the molecular frame $(\xi, \eta, \zeta)$ and the interface-fixed coordinate frame $(i, j, k){ }^{47}$ The expressions for the transformation matrices differ between different authors depending on the definition of the coordinate frames used. The operator \langle\rangle denotes the molecular orientational average over all possible orientations. The quantity $\beta_{\xi \eta \zeta}^{(2)}(\xi, \eta, \zeta=a, b, c)$ is the hyperpolarizability of the molecules and can be calculated from second-order perturbation theory. ${ }^{34}$ When the frequencies of both the visible and the sum frequency beams are far from electronic resonances, $\beta_{\xi \eta \zeta}^{(2)}$ takes the form ${ }^{34}$

$$
\beta_{\xi \eta \zeta}^{(2)}=\frac{\beta_{\xi \eta \zeta}^{q}}{\omega_{\mathrm{IR}}-\omega_{q}+i \Gamma_{q}},
$$

where $\beta_{\xi \eta \zeta}^{q}, \omega_{q}$, and $\Gamma_{q}$ are the magnitude, frequency, and damping constant, respectively, of the $q$ th vibrational mode. ${ }^{34}$ The magnitude of $\beta_{\xi \eta \zeta}^{q}$ is directly related to the IR and Raman properties of the vibrational mode by ${ }^{34}$

$$
\beta_{\xi \eta \zeta}^{q}=-\frac{1}{2 \varepsilon_{0} \omega_{q}} \frac{\partial \alpha_{\xi \eta}^{(1)}}{\partial Q_{q}} \frac{\partial \mu_{\zeta}}{\partial Q_{q}},
$$

where $\partial \alpha_{\xi \eta}^{(1)} / \partial Q_{q}$ and $\partial \mu_{\zeta} / \partial Q_{q}$ are the (Raman) polarizability tensor and the (IR) dipole derivatives, respectively, of the $q$ th vibrational mode. $\varepsilon_{0}$ is the dielectric constant of a vacuum and $Q_{q}$ is the normal coordinate. The value of $\partial \alpha_{\xi \eta}^{(1)} / \partial Q_{q}$ and $\partial \mu_{\zeta} / \partial Q_{q}$ for different kinds of molecules can be roughly estimated from the bond additivity model, as carried out by Hirose et $a l^{49}$ and Wei et al. ${ }^{50}$ It is possible to estimate the relative magnitudes of different $\chi_{i j k}^{(2)}$ components in this way.

$L_{i i}, L_{j j}$, and $L_{k k}(i, j, k=x, y, z)$ in Eq. (1) are the Fresnel coefficients or local field factors ( $L$ factors) relating input macroscopic electric fields to the macroscopic fields at the interface. For a single interface between media 1 and 2 [Fig. $1(\mathrm{a})]$, the $L$ factors in a reflected direction are given by ${ }^{39}$

$$
\begin{aligned}
& L_{x x, 12}^{R}=1-r_{p, 12}, \\
& L_{y y, 12}^{R}=1+r_{s, 12}, \\
& L_{z z, 12}^{R}=\left(1+r_{p, 12}\right)\left(\frac{n_{1}}{n_{m}}\right)^{2},
\end{aligned}
$$

where $n_{1}$ and $n_{2}$ are the refractive indices of media 1 and 2, respectively, and $n_{m}$ is the effective refractive index of the interfacial layer. The physical meaning of $n_{m}$ has been discussed elsewhere ${ }^{50,51}$ and the value of $n_{m}$ at the interface between two media can be approximately calculated using a slab model. ${ }^{51} r_{s / p, 12}$ and $t_{s / p, 12}$ are the amplitude reflection and transmission coefficients for $s$ - and $p$-polarized lights at an interface between media 1 and 2 and are given by ${ }^{36}$

$$
\begin{aligned}
& r_{s, 12}=\frac{n_{1} \cos \theta_{1}-n_{2} \cos \theta_{2}}{n_{1} \cos \theta_{1}+n_{2} \cos \theta_{2}}, \\
& t_{s, 12}=\frac{2 n_{1} \cos \theta_{1}}{n_{1} \cos \theta_{1}+n_{2} \cos \theta_{2}}, \\
& r_{p, 12}=\frac{n_{2} \cos \theta_{1}-n_{1} \cos \theta_{2}}{n_{1} \cos \theta_{2}+n_{2} \cos \theta_{1}}, \\
& t_{p, 12}=\frac{2 n_{1} \cos \theta_{1}}{n_{1} \cos \theta_{2}+n_{2} \cos \theta_{1}} .
\end{aligned}
$$

Substituting these equations into Eq. (5) yields expressions for the $L$ factors. ${ }^{51}$ The $L$ factors for SFG signals observed in the transmitted direction $\left[\mathrm{SF}_{\mathrm{T}}\right.$, Fig. 1(a)] take the same forms as Eq. (5) but with subscripts 1 and 2 interchanged. ${ }^{39}$

It should be emphasized that no thickness related interference occurs in the scheme in Fig. 1(a) and only a reflected SFG beam and a transmitted SFG beam need to be considered. This case can be generally regarded as a typical single interface for SFG measurement at, for example, air/solid, liquid/solution, and air/liquid boundaries. However, in the scheme shown in Fig. 1(b), where a thin film (with a refractive index of $n_{2}$ ) is introduced between two media (with refractive indices of $n_{1}$ and $n_{3}$ ), two interfaces appear (i.e., the "free" interface I between media 1 and 2, and the buried interface II between media 2 and 3) and both of them will 
contribute to the SFG signals. Furthermore, when the thickness of the film is comparable to the wavelength of the pumped or emitted beams, the thickness induced interference effect will also occur. In order to include these effects, the expressions for the SFG signals can be rewritten as

$$
\begin{aligned}
I_{\mathrm{SFG}}\left(\omega_{s}\right) \propto & \mid \sum_{i} \sum_{j, k} L_{i i}^{\mathrm{I}}\left(\omega_{\mathrm{SFG}}\right) \chi_{i j k}^{(2), \mathrm{I}} L_{j j}^{\mathrm{I}}\left(\omega_{\mathrm{vis}}\right) L_{k k}^{I}\left(\omega_{\mathrm{IR}}\right) \\
& +\left.\sum_{i} \sum_{j, k} L_{i i}^{\mathrm{II}}\left(\omega_{\mathrm{SFG}}\right) \chi_{i j k}^{(2), \mathrm{II}} L_{j j}^{\mathrm{II}}\left(\omega_{\mathrm{vis}}\right) L_{k k}^{\mathrm{II}}\left(\omega_{\mathrm{IR}}\right)\right|^{2} I_{\mathrm{vis}} I_{\mathrm{IR}},
\end{aligned}
$$

where superscripts I and II denote the two interfaces in Fig. 1(b). $L$ factors for incident and output electric fields at the free interface I [Fig. 1(b)] can be obtained by introducing multiple reflection terms from the thin film into Eq. (5). ${ }^{39,45}$ We have

$$
\begin{aligned}
& L_{x x}^{I}=1-\frac{r_{p, 12}+r_{p, 23} \exp (i \Delta)}{1+r_{p, 12} r_{p, 23} \exp (i \Delta)}, \\
& L_{y y}^{I}=1+\frac{r_{s, 12}+r_{s, 23} \exp (i \Delta)}{1+r_{s, 12} r_{s, 23} \exp (i \Delta)}, \\
& L_{z z}^{I}=\left(1+\frac{r_{p, 12}+r_{p, 23} \exp (i \Delta)}{1+r_{p, 12} r_{p, 23} \exp (i \Delta)}\right)\left(\frac{n_{1}}{n_{m, 12}}\right)^{2} .
\end{aligned}
$$

The reflection and transmission coefficients for interfaces I and II in Fig. 1(b) take the same form as in Eq. (6), with appropriate changes in the subscripts. $\Delta$ is the phase factor accounting for the geometrical path difference between two neighboring successive reflected or transmitted beams in the multiple reflection process, and is given by the following expression: ${ }^{39,45}$

$$
\Delta=\left(\frac{2 \pi}{\lambda}\right) n_{2} d \cos \theta_{2}
$$

with $\lambda$ being the laser wavelength for the three beams in air, $d$ is the thickness of the thin film, and $\theta_{2}$ is the refractive angle for the three beams (i.e., visible, IR, and SFG) in medium 2. The $L$ factors shown above are functions of the wavelength and can be used to calculate the $L$ factors for all three beams. It should be mentioned here that the electric field for the visible and IR beams at interface I [Fig. 1(b)] calculated using the expression for the $L$ factors in Eq. (8) includes all multiple reflections within the thin film. The detected SFG signals contain both the directly reflected light from interface I and those transmitting into medium 2 and then multiply reflected within the thin film and traveling further in the detection direction.

Next, the $L$ factors for the buried interface II between media 2 and 3 [Fig. 1(b)] can be expressed as 39

$$
\begin{aligned}
& L_{\omega_{i}, x x}^{\mathrm{II}}=\exp \left(i \Delta_{\mathrm{II}}\right) \frac{t_{p, 12} t_{p, 23}}{1+r_{p, 12} r_{p, 23} \exp (i \Delta)}, \\
& L_{\omega_{i}, y y}^{\mathrm{II}}=\exp \left(i \Delta_{\mathrm{II}}\right) \frac{t_{s, 12} t_{s, 23}}{1+r_{s, 12} r_{s, 23} \exp (i \Delta)}, \\
& L_{\omega_{i}, z z}^{\mathrm{II}}=\exp \left(i \Delta_{\mathrm{II}}\right) \frac{t_{p, 12} t_{p, 23}}{1+r_{p, 12} r_{p, 23} \exp (i \Delta)} \frac{n_{1} n_{3}}{\left(n_{m, 23}\right)^{2}},
\end{aligned}
$$

where $\exp \left(i \Delta_{\mathrm{II}}\right)$ is an additional phase factor to account for the relative phase differences generated from the beam propagating distance with respect to the coherence point, where the SFG signal from the top interface I and that from the bottom interface II interfere with each other. The calculation of this additional phase is similar to that proposed by Lambert et al. ${ }^{31}$ The expressions for $\Delta_{\mathrm{II}}$ for all three beams in copropagating incident beam geometry are as follows:

$$
\begin{aligned}
\Delta_{\mathrm{II}}(\mathrm{SF})= & \frac{2 \pi n_{2, \mathrm{SF}}}{\lambda_{\mathrm{SF}}} \frac{d}{\cos \theta_{2, \mathrm{SFG}}}, \\
\Delta_{\mathrm{II}}(\mathrm{vis})= & \frac{2 \pi n_{2, \mathrm{vis}}}{\lambda_{\mathrm{vis}}} \frac{d}{\cos \theta_{2, \mathrm{vis}}}-\frac{2 \pi n_{1, \mathrm{vis}}}{\lambda_{\mathrm{vis}}} d\left(\tan \theta_{2, \mathrm{SF}}\right. \\
& \left.+\tan \theta_{2, \mathrm{vis}}\right) \sin \theta_{1, \mathrm{vis}}, \\
\Delta_{\mathrm{II}}(\mathrm{IR})= & \frac{2 \pi n_{2, \mathrm{IR}}}{\lambda_{\mathrm{IR}}} \frac{d}{\cos \theta_{2, \mathrm{IR}}}-\frac{2 \pi n_{1, \mathrm{IR}}}{\lambda_{\mathrm{IR}}} d\left(\tan \theta_{2, \mathrm{SF}}\right. \\
& \left.+\tan \theta_{2, \mathrm{IR}}\right) \sin \theta_{1, \mathrm{IR}} .
\end{aligned}
$$

It should be noted that the equations above can be used to calculate SFG signals from thin-film systems when the thickness is less than tens of micron. The equations become inaccurate when the thickness of the thin film is comparable to or thicker than the diameter of the beam intersecting the interface.

Based on the $L$ factors and the $\chi_{i j k}^{(2)}$ given above, it is then possible to simulate SFG spectra that are useful for prediction. The SFG spectral intensities from the azimuthally isotropic monolayer with different input and output polarization combinations (ssp, sps, and $p p p$ ) can be expressed as

$$
\begin{aligned}
I_{s s p} & \propto\left|L_{y y}^{\mathrm{I}}\left(\omega_{\mathrm{SFG}}\right) L_{y y}^{\mathrm{I}}\left(\omega_{\mathrm{vis}}\right) L_{z z}^{\mathrm{I}}\left(\omega_{\mathrm{IR}}\right) \sin \theta_{\mathrm{IR}} \chi_{y y z}^{(2), \mathrm{I}}+L_{y y}^{\mathrm{II}}\left(\omega_{\mathrm{SFG}}\right) L_{y y}^{\mathrm{II}}\left(\omega_{\mathrm{vis}}\right) L_{z z}^{\mathrm{II}}\left(\omega_{\mathrm{IR}}\right) \sin \theta_{\mathrm{IR}} \chi_{y y z}^{(2), \mathrm{II}}\right|^{2} \\
& =\left|L_{y y z}^{\mathrm{I}} \sin \theta_{\mathrm{IR}} \chi_{y y z}^{(2), \mathrm{I}}+L_{y y z}^{\mathrm{II}} \sin \theta_{\mathrm{IR}} \chi_{y y z}^{(2), \mathrm{II}}\right|^{2}, \\
I_{s p s} & \propto\left|L_{y y}^{\mathrm{I}}\left(\omega_{\mathrm{SFG}}\right) L_{z z}^{\mathrm{I}}\left(\omega_{\mathrm{vis}}\right) L_{y y}^{\mathrm{I}}\left(\omega_{\mathrm{IR}}\right) \sin \theta_{\mathrm{vis}} \chi_{y z y}^{(2), \mathrm{I}}+L_{y y}^{\mathrm{II}}\left(\omega_{\mathrm{SFG}}\right) L_{z z}^{\mathrm{II}}\left(\omega_{\mathrm{vis}}\right) L_{y y}^{\mathrm{II}}\left(\omega_{\mathrm{IR}}\right) \sin \theta_{\mathrm{vis}} \chi_{y z y}^{(2), \mathrm{II}}\right|^{2} \\
& =\left|L_{y z y}^{\mathrm{I}} \sin \theta_{\mathrm{vis}} \chi_{y z y}^{(2), \mathrm{I}}+L_{y z y}^{\mathrm{II}} \sin \theta_{\mathrm{vis}} \chi_{y z y}^{(2), \mathrm{II}}\right|^{2},
\end{aligned}
$$




$$
\begin{aligned}
I_{p p p} \propto & \mid-L_{x x}^{\mathrm{I}}\left(\omega_{\mathrm{SFG}}\right) L_{x x}^{\mathrm{I}}\left(\omega_{\mathrm{vis}}\right) L_{z z}^{\mathrm{I}}\left(\omega_{\mathrm{IR}}\right) \cos \theta_{\mathrm{SFG}} \cos \theta_{\mathrm{vis}} \sin \theta_{\mathrm{IR}} \chi_{x x z}^{(2), \mathrm{I}}-L_{x x}^{\mathrm{I}}\left(\omega_{\mathrm{SFG}}\right) L_{z z}^{\mathrm{I}}\left(\omega_{\mathrm{vis}}\right) L_{x x}^{\mathrm{I}}\left(\omega_{\mathrm{IR}}\right) \cos \theta_{\mathrm{SFG}} \sin \theta_{\mathrm{vis}} \cos \theta_{\mathrm{IR}} \chi_{x z x}^{(2), \mathrm{I}} \\
& +L_{z z}^{\mathrm{I}}\left(\omega_{\mathrm{SFG}}\right) L_{x x}^{E}\left(\omega_{\mathrm{vis}}\right) L_{x x}^{\mathrm{I}}\left(\omega_{\mathrm{IR}}\right) \sin \theta_{\mathrm{SFG}} \cos \theta_{\mathrm{vis}} \cos \theta_{\mathrm{IR}} \chi_{z x x}^{(2), \mathrm{I}}+L_{z z}^{\mathrm{I}}\left(\omega_{\mathrm{SFG}}\right) L_{z z}^{\mathrm{I}}\left(\omega_{\mathrm{vis}}\right) L_{z z}^{\mathrm{I}}\left(\omega_{\mathrm{IR}}\right) \sin \theta_{\mathrm{SFG}} \sin \theta_{\mathrm{vis}} \sin \theta_{\mathrm{IR}} \chi_{z z z}^{(2), \mathrm{I}} \\
& -L_{x x}^{\mathrm{II}}\left(\omega_{\mathrm{SFG}}\right) L_{x x}^{\mathrm{II}}\left(\omega_{\mathrm{vis}}\right) L_{z z}^{\mathrm{II}}\left(\omega_{\mathrm{IR}}\right) \cos \theta_{\mathrm{SFG}} \cos \theta_{\mathrm{vis}} \sin \theta_{\mathrm{IR}} \chi_{x x z}^{(2), \mathrm{II}}-L_{x x}^{\mathrm{II}}\left(\omega_{\mathrm{SFG}}\right) L_{z z}^{\mathrm{II}}\left(\omega_{\mathrm{vis}}\right) L_{x x}^{\mathrm{II}}\left(\omega_{\mathrm{IR}}\right) \cos \theta_{\mathrm{SFG}} \sin \theta_{\mathrm{vis}} \cos \theta_{\mathrm{IR}} \chi_{x z x}^{(2), \mathrm{II}} \\
& +L_{z z}^{\mathrm{II}}\left(\omega_{\mathrm{SFG}}\right) L_{x x}^{\mathrm{II}}\left(\omega_{\mathrm{vis}}\right) L_{x x}^{\mathrm{II}}\left(\omega_{\mathrm{IR}}\right) \sin \theta_{\mathrm{SFG}} \cos \theta_{\mathrm{vis}} \cos \theta_{\mathrm{IR}} \chi_{z x x}^{(2), \mathrm{II}}+\left.L_{z z}^{\mathrm{II}}\left(\omega_{\mathrm{SFG}}\right) L_{z z}^{\mathrm{II}}\left(\omega_{\mathrm{vis}}\right) L_{z z}^{\mathrm{II}}\left(\omega_{\mathrm{IR}}\right) \sin \theta_{\mathrm{SFG}} \sin \theta_{\mathrm{vis}} \sin \theta_{\mathrm{IR}} \chi_{z z z}^{(2), \mathrm{II}}\right|^{2} \\
= & -L_{x x z}^{\mathrm{I}} \cos \theta_{\mathrm{SFG}} \cos \theta_{\mathrm{vis}} \sin \theta_{\mathrm{IR}} \chi_{x x z}^{(2), \mathrm{I}}-L_{x z x}^{\mathrm{I}} \cos \theta_{\mathrm{SFG}} \sin \theta_{\mathrm{vis}} \cos \theta_{\mathrm{IR}} \chi_{x z x}^{(2), \mathrm{I}}+L_{z x x}^{\mathrm{I}} \sin \theta_{\mathrm{SFG}} \cos \theta_{\mathrm{vis}} \cos \theta_{\mathrm{IR}} \chi_{z x x}^{(2), \mathrm{I}} \\
& +L_{z z z}^{\mathrm{I}} \sin \theta_{\mathrm{SFG}} \sin \theta_{\mathrm{vis}} \sin \theta_{\mathrm{IR}} \chi_{z z z}^{(2), \mathrm{I}}-L_{x x z}^{\mathrm{II}} \cos \theta_{\mathrm{SFG}} \cos \theta_{\mathrm{vis}} \sin \theta_{\mathrm{IR}} \chi_{x x z}^{(2), \mathrm{II}}-L_{x z x}^{\mathrm{II}} \cos \theta_{\mathrm{SFG}} \sin \theta_{\mathrm{vis}} \cos \theta_{\mathrm{IR}} \chi_{x z x}^{(2), \mathrm{II}} \\
& +L_{z x x}^{\mathrm{II}} \sin \theta_{\mathrm{SFG}} \cos \theta_{\mathrm{vis}} \cos \theta_{\mathrm{IR}} \chi_{z x x}^{(2), \mathrm{II}}+\left.L_{z z z}^{\mathrm{II}} \sin \theta_{\mathrm{SFG}} \sin \theta_{\mathrm{vis}} \sin \theta_{I R} \chi_{z z z}^{(2), \mathrm{II}}\right|^{2} .
\end{aligned}
$$

The $L$ factors in Eq. (12) have been defined above and are functions of parameters such as film thickness, refractive indices, and incident beam angles and can be calculated once the optical geometry has been selected. On the other hand, the susceptibility parameters only depend on the structures of the species at the interface. Once all of these conditions are known, the SFG spectra at different thicknesses can be simulated. It should be noted that the signs in Eq. (12) are for the copropagating incident beam geometry. The signs for the second, third, sixth, and seventh terms in Eq. (12c) are reversed if a counterpropagating incident beam geometry is used.

\section{MODEL: MONOLAYER/THIN DIELECTRIC FILM/ METAL SUBSTRATE}

\section{A. Model description}

The model employed for simulating SFG spectra in the present paper is shown schematically in Fig. 2. A wellordered monolayer of aliphatic fatty acid with a long alkyl chain (for example, arachidic acid) is deposited on a variable thickness dielectric thin film which is in contact with a gold substrate. Assuming that the dielectric film itself exhibits no resonances in the $\mathrm{C}-\mathrm{H}$ stretching region as well as no nonresonant signals, the only resonant SFG signal in the $\mathrm{C}-\mathrm{H}$ region will arise from the fatty acid monolayer. Furthermore, the buried interface will only give nonresonant SFG signal from the gold substrate. This kind of thin-film model can be

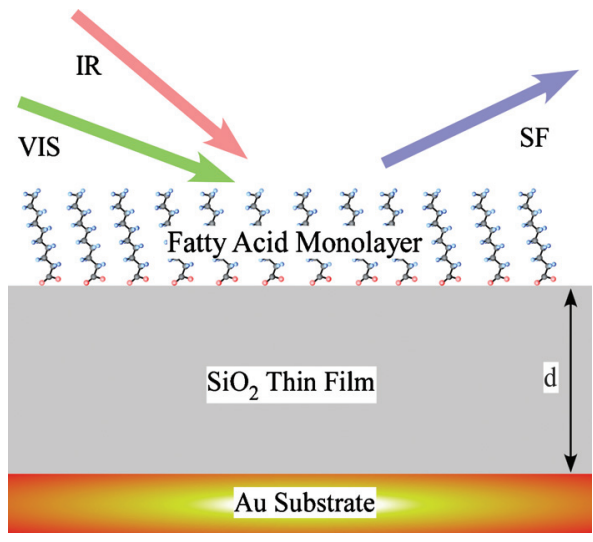

FIG. 2. Schematic of the air/fatty acid monolayer/SiO $/ \mathrm{S}_{2}$ thin-film/gold substrate model of a thin-film system. experimentally reproduced by using deuterated LB multilayers to verify the validity of the calculations and this will be reported in the following paper. ${ }^{46}$

In Secs. III B and III C, we first elucidate the thickness dependence of the $L$ factors for the two interfaces (buried and free interfaces, Figs. 3 and 4) at a constant IR frequency. Then, we will show the IR frequency dependent properties of the susceptibilities of the terminal $\mathrm{CH}_{3}$ group in the hydrocarbon chains of the well-ordered monolayer at the air/thinfilm interface. Next, we will present our simulation results for SFG spectra of the $\mathrm{CH}_{3}$ group in the model thin-film system based on the $L$ factor and susceptibility calculations. Finally, we will propose a method to predict the spectral line shapes for different film thicknesses.

\section{B. Thickness dependence of the $L$ factors \\ 1. $L$ factors for the buried film/gold interface}

As described in Sec. II, in order to understand how the thickness factor affects the SFG signal, one needs to calculate the electric field at each interface, which is contained in the $L$ factors. The wavelength of the visible and IR beams are fixed at 800 and $3125 \mathrm{~nm}$ (i.e., $3200 \mathrm{~cm}^{-1}$ ), respectively, hence $\mathrm{SFG}$ occurs at $650 \mathrm{~nm}$. Figure 3 shows the calculated results for the moduli of the combined $L$ factor, $L_{i j k}$ (solid trace) and its three components, i.e., $L_{i i}$ (SFG, red-dashed trace), $L_{j j}$ (visible, blue-dotted trace), and $L_{k k}$ (IR, purpledotted-dashed trace), at the buried interface between the dielectric thin film and the gold substrate in the model system (Fig. 2), as a function of the dielectric film thickness increasing from 0 to $600 \mathrm{~nm}$. $L_{y y z}$ [solid trace, Fig. 3(a)], $L_{y z y}$ [solid trace, Fig. 3(b)], and $L_{z z z}$ [solid trace, Fig. 3(c)] corresponding to the $L$ factors for the ssp, sps, and ppp (Ref. 52) polarization combinations, respectively. The moduli of these combined $L$ factors as a function of film thickness will contribute to the background signal of the net SFG spectra.

It was found that the combined $L$ factors (i.e., $L_{i j k}$ ) of all three polarization combinations vary significantly with the film thickness, but no clear periodicities were observed. A comparison of results for $L_{y y z}, L_{y z y}$, and $L_{z z z}$ [Figs. 3(a)-3(c)] shows that the thickness dependences of the $L$ factors for different polarization combinations are totally different. The magnitude of $L_{z z z}$ is much higher than that of $L_{y y z}$ or $L_{y z y}$, at any film thickness. In fact, this is one of the reasons that normally, a nonresonant background from a bare gold sub- 
(a)

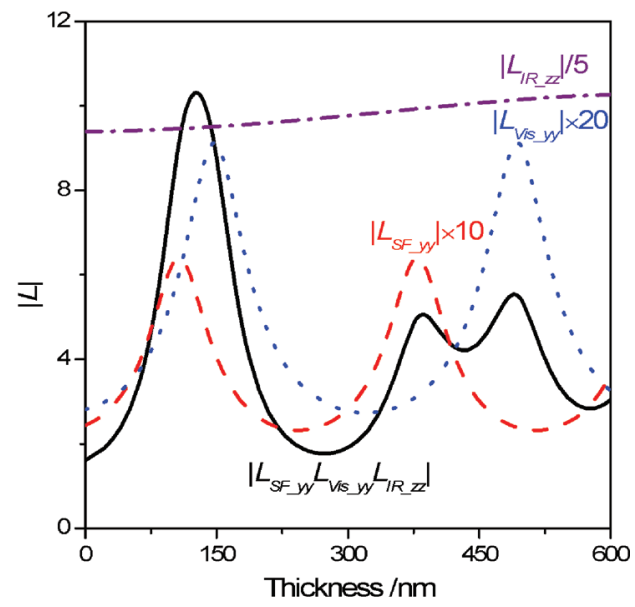

(b)

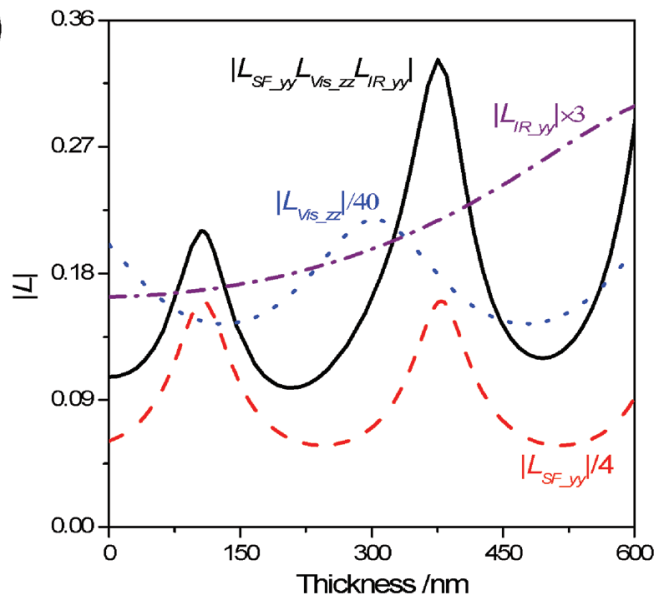

(c)

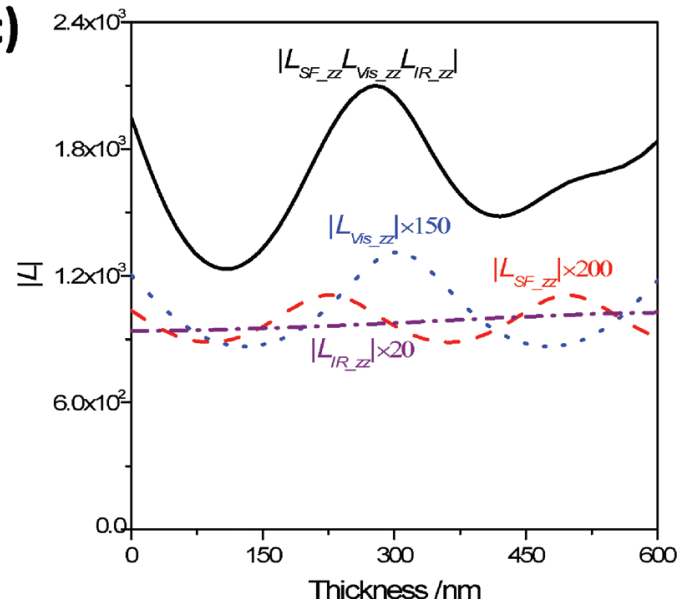

FIG. 3. Simulated results of the modulus of the combined Fresnel factors $L_{i j k}$ (solid line) and their components, $L_{\mathrm{SFG}}$ (red-dashed trace), $L_{\mathrm{vis}}$ (bluedotted trace), and $L_{\mathrm{IR}}$ (purple-dotted-dashed trace) calculated according to the thin-film model in Fig. 2 for the buried thin-film/gold interface with (a) ssp-, (b) sps-, and (c) ppp-polarization combinations. For clarity the intensity of the traces has been multiplied by the factors shown.

strate or gold surface modified by an organic monolayer can only be easily observed in the $p p p$-polarization in contrast to the ssp- and sps-polarizations.

The polarization dependent features of the combined $L$ factors of Fig. 3 are related to the features of their individual components. For example, the combined $L$ factor $L_{y y z}$ shows the first maximum value around $125 \mathrm{~nm}$ [Fig. 3(a)], which is contributed by the $L$ factor of both the SFG component (a)

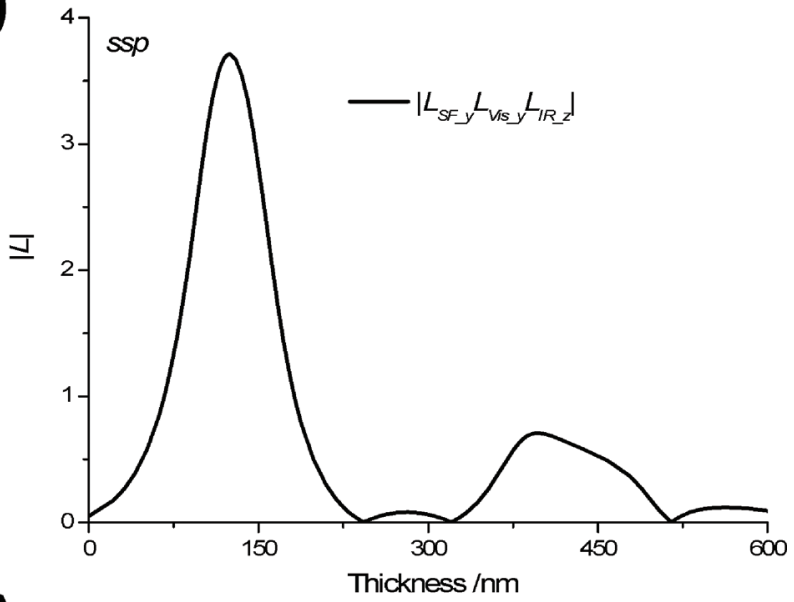

(b)

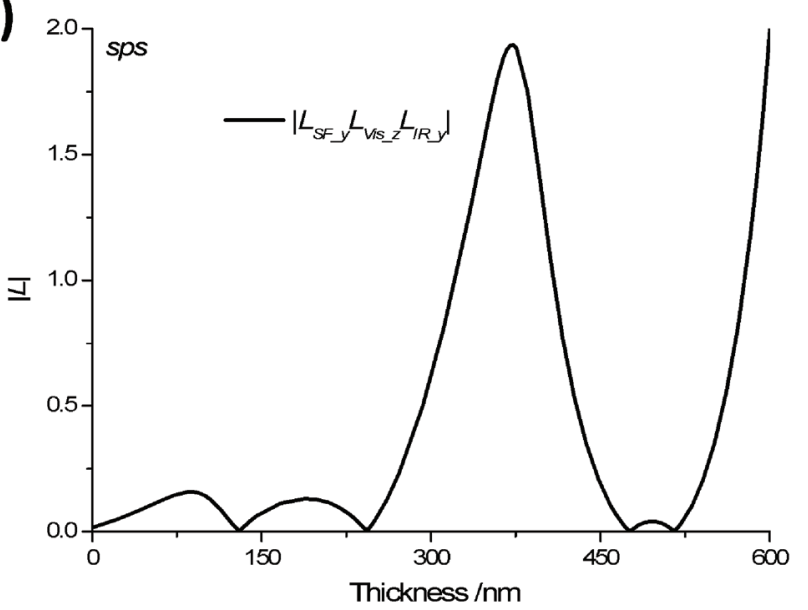

(c)

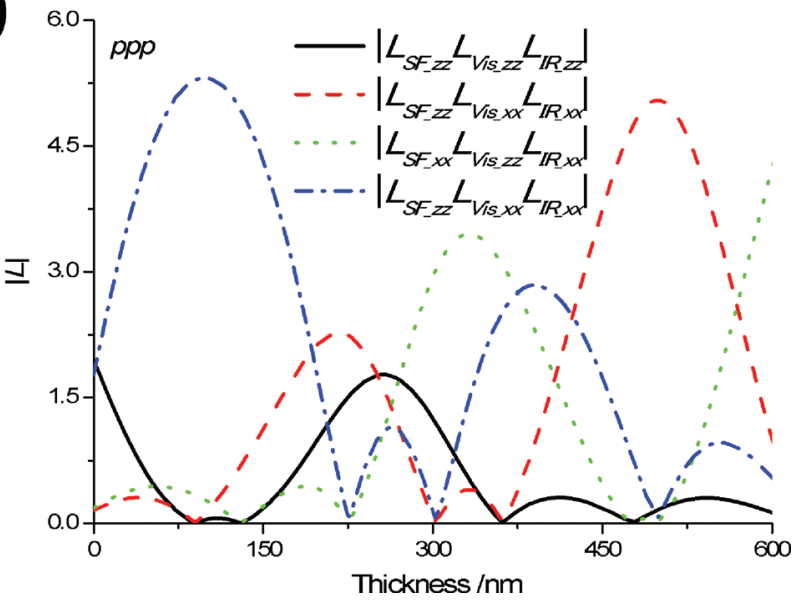

FIG. 4. Simulated results of the modulus of the combined Fresnel factors $L_{i j k}$ (solid line) calculated according to the thin-film model in Fig. 2 for the free air/thin-film interface with (a) $s s p-$, (b) $s p s-$, and (c) ppp-polarization combinations.

[dashed trace, Fig. 3(a)] and the visible component [dotted trace, Fig. 3(a)]. The subsequent small maxima at 385 and $490 \mathrm{~nm}$ are due to the $L$ factors of the SFG component and the visible component, respectively, acting separately.

In order to understand these complicated features, it is instructive to examine the behavior of each component of the $L$ factor. As shown in Fig. 3, although the combined $L$ factors $\left(L_{i j k}\right)$ exhibit no clear periodicity, the modulus of each component $\left(L_{\mathrm{SFG}}, L_{\mathrm{vis}}\right.$, or $\left.L_{\mathrm{IR}}\right)$ does oscillate periodically with the film thickness. For different beam components under the 
same Cartesian axes, e.g., $L_{z z, \mathrm{SFG}}$ [Fig. 3(c), dashed traces], $L_{z z, \text { vis }}$ [Fig. 3(c), dotted traces], and $L_{z z, \text { IR }}$ [Fig. 3(c), dasheddotted traces], the periodicities are different $(272,344$, and $1320 \mathrm{~nm}$ for SFG, visible, and IR components, respectively.) The results are only shown up to thicknesses of $600 \mathrm{~nm}$ in the present figure. Consequently, $L_{i j k}$ (solid trace, Fig. 3), is more strongly affected by features of the SFG and visible beams in comparison with that of the IR beam for the thickness scale shown. Alternatively, for the same beam component under different Cartesian axes, e.g., $L_{y y, \text { vis }}$ [Fig. 3(a), blue-dotted trace] and $L_{z z \text {,vis }}$ [Fig. 3(b), blue-dotted trace], the periodicities are the same, while the intensities as well as the shapes are different. The modulus of $L_{z z \text {,vis }}$ shows much higher intensity and oscillates with much higher amplitude than that of $L_{y y \text {,vis }}$. The reason is due to the imaginary refractive index of the gold substrate. The incident electric field along the $z$ Cartesian axis will constructively merge with the reflected beam while that along the $y$ axis cancels with the reflecting beam. ${ }^{35}$

It is worth noting that in the Lambert et al. ${ }^{31}$ model, the $L$ factors for the nonresonant signal were not considered. As we have shown here though, the nonresonant signal also changes significantly with the film thickness through the $L$ factor and must be taken into account. Moreover, as will be demonstrated later, the intensity of the nonresonant signal makes an important contribution to the spectral features.

The $L_{z z z}$ component plays a dominant role in the ppp-polarized SFG spectrum for the organic thin-film/gold interface in comparison with the other three $L$ factors. This is only true on metal substrate interfaces such as gold and silver but not on dielectric substrate interfaces with lower nonresonant backgrounds, in which the other three components of the $L$ factors for ppp-polarization will also give reasonable contributions in comparison to $L_{z z z}$.

\section{2. $L$ factors for the free air/film interface}

Figure 4 shows the calculated moduli of (a) $L_{y y z}$, (b) $L_{y z y}$, (c) $L_{x x z}, L_{x z x}, L_{z x x}$, and $L_{z z z}$ for the free air/film interface for the same model system (Fig. 2) using Eq. (10) as a function of film thickness $(0-600 \mathrm{~nm})$. Several different features arise in comparison with those from the buried film/gold interface (Fig. 3). The modulus maxima of each $L$ factor on the free interface are in the same order for each polarization combination as those of the buried interface (Fig. 3). It is interesting to note that the modulus for $L_{z z z}$ is no longer dominant in the $p p p$-polarization combination in comparison with the other three components, $L_{x x z}, L_{x z x}$, and $L_{z x x}$. Furthermore, as shown in Fig. 4, the moduli of these $L$ factors can become zero at certain film thicknesses. Such features are not observed for the buried interface (Fig. 3).

The considerable differences between the $L$ factors from the free and buried interface can be easily understood from the equations used to generate them. As has been discussed by Lambert et al. ${ }^{31}$ and Feller et al. ${ }^{39}$ the $L$ factors for the free interface [Eq. (8)] encompass two terms, one is the electric field that is directly reflected from the surface and the other is the electric field that is multiply reflecting within the thin film. The magnitudes of the two terms are comparable to each other while the relative phases between the two terms oscillate periodically with the film thickness. Consequently, as the film thickness increases, constructive and destructive coherences will occur between the two terms, where the former will result in a maximum value for the moduli of the $L$ factors, while the latter will result in a zero point for the moduli of the $L$ factors. As a result, the combined $L$ factors show zero values at certain thicknesses. The properties of the $L$ factors at the free air/film interface are consistent with those reported by Wilson et $a l^{40}$ from an air/polymer/ $/ \mathrm{SiO}_{2} /$ gold thin-film sample. For example, the features of their simulation results and ours are similar to each other if the thickness of the polymer in their study is taken into consideration. Specifically, they also observed that the combined $L$ factors of the air/polymer interface at certain thicknesses (e.g., at $\mathrm{SiO}_{2}$ thickness of $\sim 70,130$, and 325 $\mathrm{nm})$ can drop to zero.

\section{Susceptibility of the terminal $\mathrm{CH}_{3}$ group in the model system}

According to the model described above (Fig. 2), the only SFG resonant sources in the $\mathrm{C}-\mathrm{H}$ stretching region are the terminal $\mathrm{CH}_{3}$ groups in the hydrocarbon chains of the well-ordered monolayer at the air/thin-film interface. ${ }^{16}$ The methylene $\left(\mathrm{CH}_{2}\right)$ group resonances in the monolayer are usually relatively weak ${ }^{16}$ and are thus omitted in the present study. The second-order nonlinear susceptibility equations for the symmetric $\left(\mathrm{r}^{+}\right)$and asymmetric $\left(\mathrm{r}^{-}\right)$vibrational modes of the $\mathrm{CH}_{3}$ group have been published elsewhere. ${ }^{31,53}$ The Fermi resonance $\left(\mathrm{r}_{\mathrm{FR}}^{+}\right)$mode, which is usually observed for the $\mathrm{CH}_{3}$ group in molecules with long alkyl chains, is modeled with identical equations to those used for the symmetric mode, as proposed by Lambert et al. ${ }^{31}$ For convenience the equations for the $\mathrm{r}$ vibrational modes are reproduced here.

(a) $\mathrm{r}^{+}$and $\mathrm{r}_{\mathrm{FR}}^{+}$modes,

$$
\begin{aligned}
& \chi_{z z z}^{(2)}=N_{s} \beta_{c c c}^{(2)}\left[R\langle\cos \theta\rangle+\left\langle\cos ^{3} \theta\right\rangle(1-R)\right\rfloor, \\
& \chi_{x x z}^{(2)} \equiv \chi_{y y z}^{(2)}=\frac{1}{2} N_{s} \beta_{c c c}^{(2)}\left[\langle\cos \theta\rangle(1+R)-\left\langle\cos ^{3} \theta\right\rangle(1-R)\right],
\end{aligned}
$$

$$
\begin{aligned}
\chi_{x z x}^{(2)} \equiv & \chi_{y z y}^{(2)} \equiv \chi_{z x x}^{(2)} \equiv \chi_{z y y}^{(2)}=\frac{1}{2} N_{s} \beta_{c c c}^{(2)}(1-R)[\langle\cos \theta\rangle \\
& \left.-\left\langle\cos ^{3} \theta\right\rangle\right] .
\end{aligned}
$$

(b) $\mathrm{r}^{-}$mode,

$$
\begin{aligned}
& \chi_{z z z}^{(2)}=2 N_{s} \beta_{a c a}^{(2)}\left(\langle\cos \theta\rangle-\left\langle\cos ^{3} \theta\right\rangle\right), \\
& \chi_{x x z}^{(2)} \equiv \chi_{y y z}^{(2)}=-N_{s} \beta_{a c a}^{(2)}\left(\langle\cos \theta\rangle-\left\langle\cos ^{3} \theta\right\rangle\right), \\
& \chi_{x z x}^{(2)} \equiv \chi_{y z y}^{(2)} \equiv \chi_{z x x}^{(2)} \equiv \chi_{z y y}^{(2)}=N_{s} \beta_{a c a}^{(2)}\left\langle\cos ^{3} \theta\right\rangle,
\end{aligned}
$$

where $N_{s}$ is the number of arachidate molecules at the interface and considered as constant in the calculation and therefore neglected; $\beta_{c c c}^{(2)}$ and $\beta_{a c a}^{(2)}$ are the hyperpolarizabilities of the $\mathrm{CH}_{3}$ groups and take the same form as Eq. (3). The hyperpolarizability ratio factor $R$ in Eq. (13) is reported to be in the range of 2.0-4.0 for molecules with long alkyl chains. ${ }^{31,51,54} \theta$ is the angle of the $c$ symmetry axis of the terminal $\mathrm{CH}_{3}$ group with respect to the surface normal (and 
TABLE I. Parameters used for the SFG spectral simulations.

\begin{tabular}{lccccc}
\hline \hline Parameter & Value & Parameter & Value & Parameter & Value \\
\hline$\theta_{1, \text { vis }}$ & $70^{\circ}$ & $R$ & 1.9 & $\left|\chi_{\mathrm{NR}}^{(2)}\right|_{z z z}$ & 0.0001 \\
$\theta_{1, \mathrm{IR}}$ & $50^{\circ}$ & $\theta$ & $19^{\circ}$ & $\left|\chi_{\mathrm{NR}}^{(2)}\right|_{y y z}$ & 0.007 \\
$\lambda_{\text {vis }}$ & $800 \mathrm{~nm}$ & $n_{2, \mathrm{SF}}$ & 1.5 & $\mid \chi_{\mathrm{NNR}}^{(2)}$ & 0.01 \\
$\omega_{\mathrm{IR}}$ & $2800-3000 \mathrm{~cm}^{-1}$ & $n_{2, \mathrm{vis}}$ & 1.49 & $\varepsilon_{\mathrm{NR}, z z z}$ & $80^{\circ}$ \\
$\omega_{r+}$ & $2878 \mathrm{~cm}^{-1}$ & $n_{2, \mathrm{IR}}$ & 1.45 & $\varepsilon_{\mathrm{NR}, y y z}$ & $130^{\circ}$ \\
$\omega_{\mathrm{FR}}$ & $2940 \mathrm{~cm}^{-1}$ & $n_{3, \mathrm{SF}}$ & $0.12+3.29 i$ & $\varepsilon_{\mathrm{NR}, y z y}$ & $240^{\circ}$ \\
$\omega_{r-}$ & $2965 \mathrm{~cm}^{-1}$ & $n_{3, \mathrm{vis}}$ & $0.08+4.78 i$ & $n_{m, 12}$ & 1.2 \\
$\Gamma$ & $5 \mathrm{~cm}^{-1}$ & $n_{3, \mathrm{IR}}$ & $0.69+22.63 i$ & $n_{m, 23}$ & $1^{\mathrm{a}}$ \\
\hline \hline
\end{tabular}

${ }^{\mathrm{a}}$ The effective refractive index of the sample/gold interface is attributed to the nonresonant susceptibility component.

is not to be confused with the incident angles for the laser beams used in earlier equations.) The operator \langle\rangle carries out an ensemble average of the orientation angle via the distribution function ${ }^{50}$

$$
\left\langle\cos ^{n} \theta\right\rangle=\int_{-1}^{1} \cos ^{n} \theta f(\cos \theta) d\left(\cos ^{n} \theta\right) .
$$

Here $f(\cos \theta)$ denotes the distribution function, which can be expressed in Gaussian or maximum entropy form. ${ }^{50}$ In the present study, the Gaussian form is employed, ${ }^{50}$

$$
f(\theta)=\frac{1}{\sqrt{2 \pi} \sigma} \exp \left[-\frac{\left(\theta-\theta_{0}\right)^{2}}{2 \sigma^{2}}\right]
$$

where $\theta_{0}$ is the mean orientation angle; $\sigma$ is the root-meansquare width. In the case of a $\delta$-distribution, $\sigma=0^{\circ}$.

The optical parameters used in the simulation are summarized in Table I. The refractive indices of the media are available from the literature. ${ }^{55}$ The effective refractive index $n_{m}$ of the interfacial layer at the air/film interface was determined to be $1.2 \pm 0.1$ for the present thin-film model calculated using the slab model reported by Zhuang et al. ${ }^{51}$ The modulus and phase $(\varepsilon)$ of the nonresonant susceptibility component have been obtained by comparing the simulated spectrum with the observed one from the film at a certain thickness. $^{31}$

The simulation was carried out using a numerical calculation program (MAPLE $12^{\circledR}$, Maplesoft, Waterloo, Canada). All three $\mathrm{CH}_{3}$ vibrational modes, with frequencies set at 2878, 2940, and $2965 \mathrm{~cm}^{-1}$, were simulated simultaneously for different film thicknesses as a function of the IR frequency $\left(2800-3000 \mathrm{~cm}^{-1}\right)$.

The susceptibility tensors $\chi_{y y z}^{(2)}\left(=\chi_{x x z}^{(2)}\right), \chi_{y z y}^{(2)}\left(=\chi_{x z x}^{(2)}=\chi_{z x x}^{(2)}\right)$, and $\chi_{z z z}^{(2)}$ for the symmetric, Fermi resonance, and asymmetric modes of the terminal $\mathrm{CH}_{3}$ groups were calculated using Eqs. (13) and (14) and their moduli are shown in Fig. 5 as a function of the IR frequency between 2800 and $3000 \mathrm{~cm}^{-1}$. The "spectrum" of $\chi_{y y z}^{(2)}\left(=\chi_{x x z}^{(2)}\right.$ is dominated by the symmetric $\left(\mathrm{r}^{+}\right)$and Fermi resonance modes, while that of $\chi_{y z y}^{(2)}$ $\times\left(=\chi_{x z x}^{(2)}=\chi_{z x x}^{(2)}\right)$ is dominated by the asymmetric $\left(\mathrm{r}^{-}\right)$mode. All three resonant modes are significant for the $\chi_{z z z}^{(2)}$ susceptibility. As mentioned above, $\chi_{y y z}^{(2)}$ and $\chi_{y z y}^{(2)}$ are the main susceptibility terms in ssp- and sps-polarized SFG spectra, respectively, while $\chi_{z z z}^{(2)}, \chi_{x x z}^{(2)}, \chi_{x z x}^{(2)}$, and $\chi_{z x x}^{(2)}$ are the four $\chi_{i j k}^{(2)}$ components contributing to the $p p p$-polarized SFG spectra
[Eq. (12c)]. It should be emphasized here that these simulated SFG spectra (i.e., nonlinear susceptibilities $\chi^{(2)}$ ) are not influenced by the $L$ factors relating to the optical geometry and film thickness and therefore are different from the experimentally observed SFG spectra.

As discussed above, the SFG spectral profiles and intensities are significantly affected by interference between the $L$ factors and the susceptibilities from different interfaces in the system. In Sec. IV, we will first simulate SFG spectra with different polarization combinations for the thin-film model systems shown in Fig. 2 based on the general theoretical model given above.

\section{SFG SPECTRAL FEATURES FOR THE $\mathrm{CH}_{3}$ GROUP IN THE MODEL SYSTEM}

\section{A. ssp-polarization}

Figure 6(a) shows the simulated ssp-polarized SFG spectra in the $\mathrm{C}-\mathrm{H}$ stretching region for the model system (Fig. 2). This figure clearly demonstrates that the spectral shape and intensities change significantly with the film thickness. Figure 6(b) shows the SFG intensities for the $\mathrm{r}^{+}$-mode at $2878 \mathrm{~cm}^{-1}$ and the base line signal contributed from the gold substrate at $3200 \mathrm{~cm}^{-1}$ as a function of film thickness. The thickness dependent intensity of the SFG signal from the $\mathrm{r}^{-}$mode is relatively weak and similar to the intensity of the

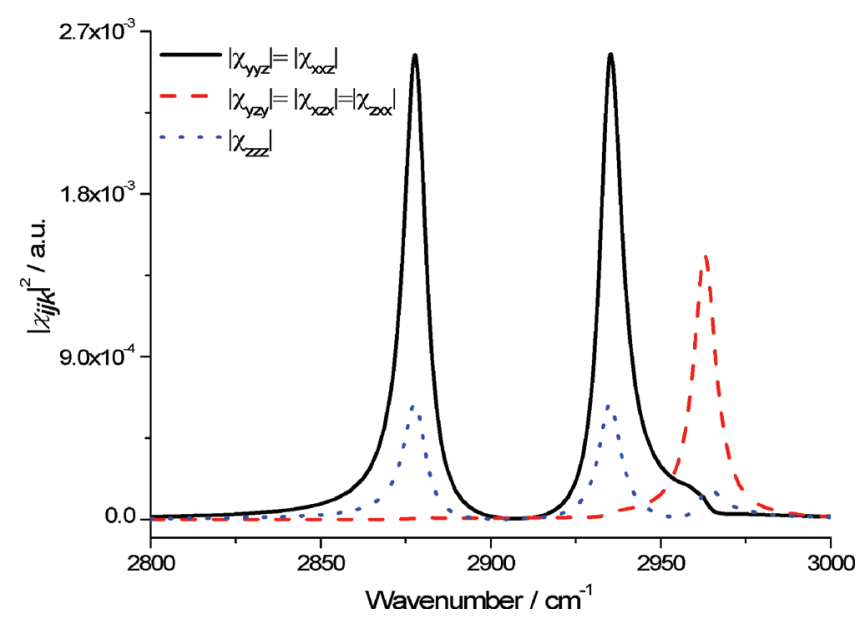

FIG. 5. Calculated susceptibilities of $\chi_{y y x}=\chi_{x x z}$ (solid), $\chi_{y z y}=\chi_{x z x}=\chi_{z x x}$ (dashed), and $\chi_{z z z}$ (dotted) for the methyl group in the IR frequency region between 2800 and $3000 \mathrm{~cm}^{-1}$. See Table I for simulation parameters. 
(a)
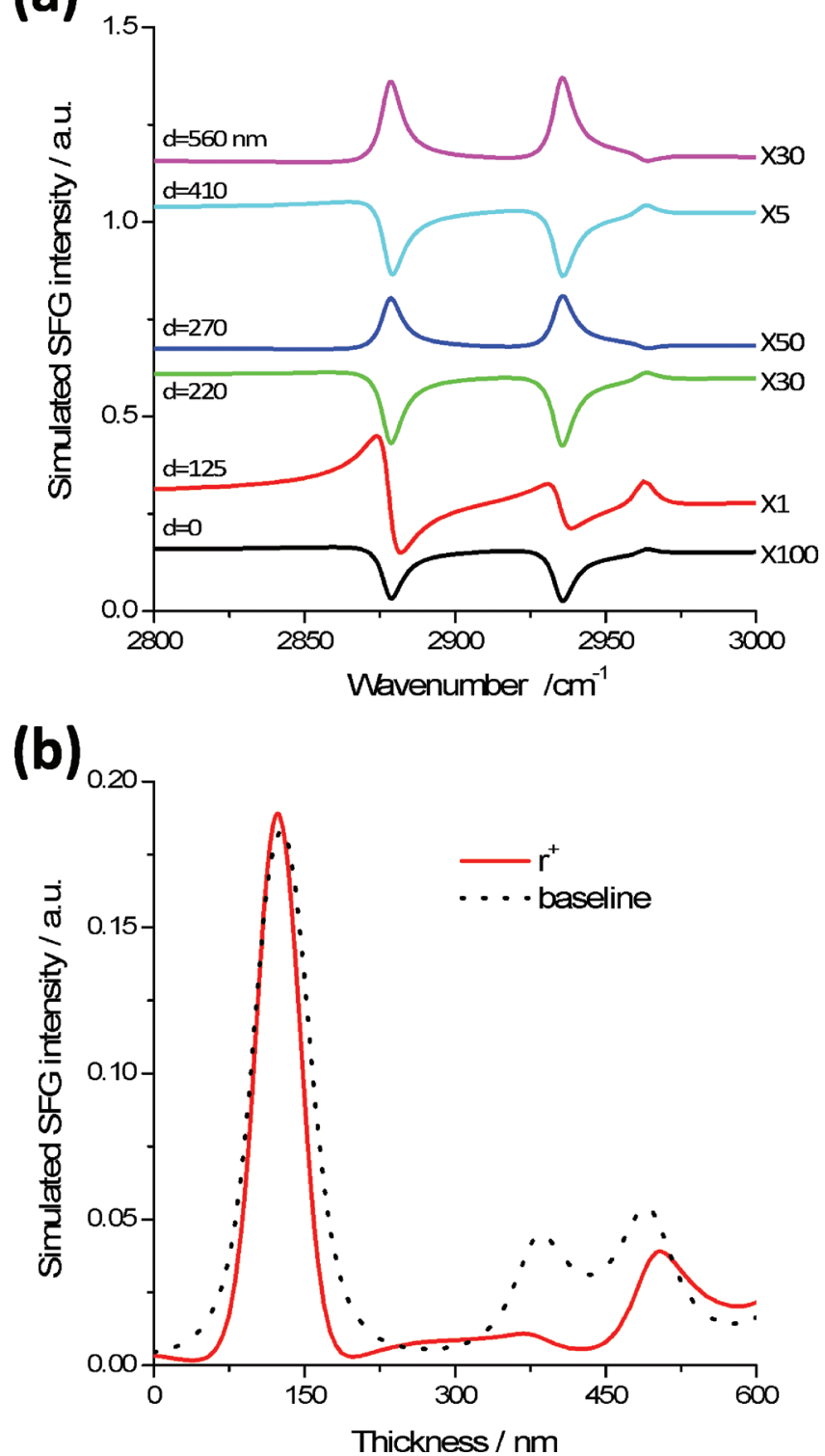

FIG. 6. Simulated results for the thickness dependence (d) (nm) of (a) SFG spectral shapes and (b) SFG intensities for the symmetric $\mathrm{C}-\mathrm{H}$ stretching mode $\left(\mathrm{r}^{+}, 2878 \mathrm{~cm}^{-1}\right)$ and base line $\left(3200 \mathrm{~cm}^{-1}\right)$ for the model system under the $s s p$-polarization combination. Base line levels for each spectrum in (a) are displaced for clarity. See text for simulation parameters.

base line signal. When the film is thin (for example, a monolayer has a film thickness of $\sim 2.5 \mathrm{~nm}$ ), the SFG signal is weak. The symmetric and Fermi resonance $\mathrm{C}-\mathrm{H}$ stretching modes appear as dips [Fig. 6(a)]. The ssp-polarized SFG intensities increase rapidly with thickness and reach the first maximum around $125 \mathrm{~nm}$, which is approximately two orders higher than that of the thinner film of $\sim 2.5 \mathrm{~nm}$. The calculation suggests that one can easily obtain an experimental SFG spectrum for this film thickness. The SFG intensity then quickly decreases with further increases in thickness. The peak intensity for the $\mathrm{r}^{+}$mode gives a smaller maximum at $505 \mathrm{~nm}$ while that for the base line shows two smaller maxima at 385 and $490 \mathrm{~nm}$. Not only the SFG spectral intensities but also the SFG spectral shapes change dramatically with thickness. As shown in Fig. 6(a), the symmetric and Fermi resonance $\mathrm{C}-\mathrm{H}$ stretching modes change from (a)

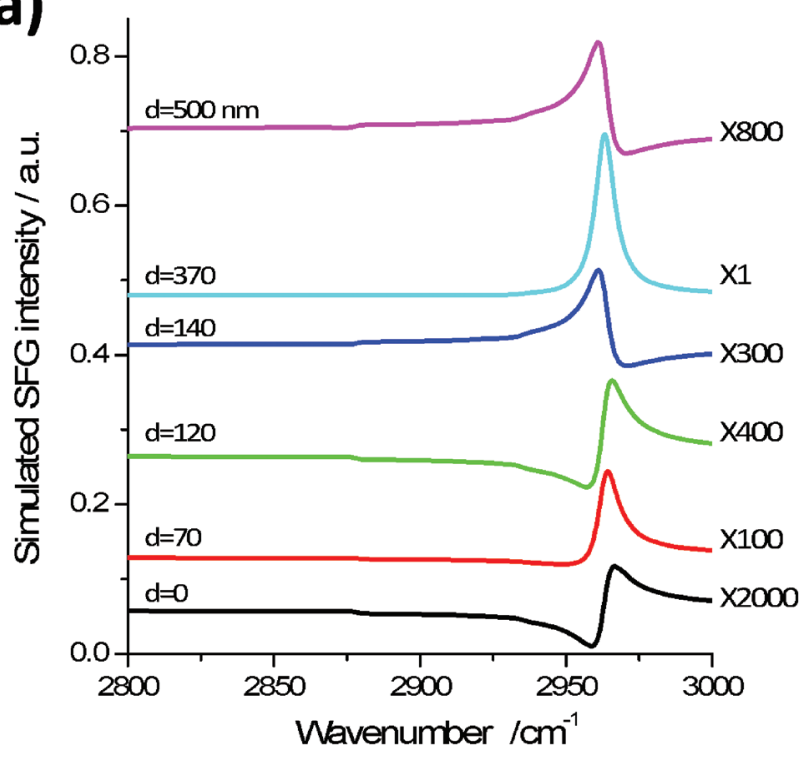

(b)

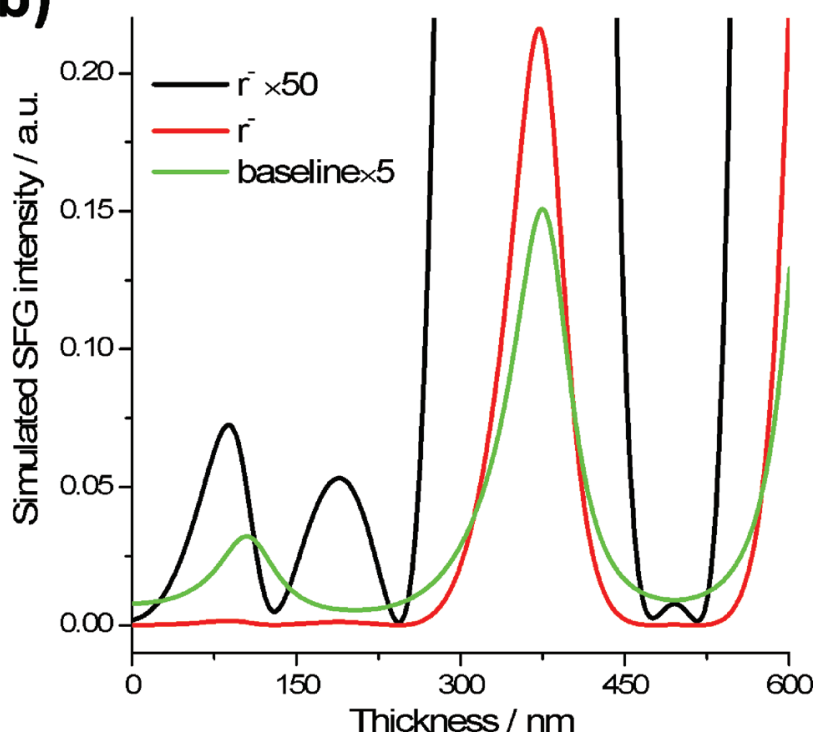

FIG. 7. Simulated results for the thickness dependence of (a) SFG spectral shapes and (b) SFG intensities for the asymmetric $\mathrm{C}-\mathrm{H}$ stretching modes $\left(\mathrm{r}^{-}, 2963 \mathrm{~cm}^{-1}\right)$ and base line (at $\left.3200 \mathrm{~cm}^{-1}\right)$ for the model system under the sps-polarization combination. In order to show the peak intensity clearly, the results for the $\mathrm{r}^{-}$mode and the base line are scaled by a factor of 50 and 5 , respectively. Base line levels for each spectrum in (a) are displaced for clarity. See text for simulation parameters.

dips (at $2.5 \mathrm{~nm}$ ) to Z-shaped bipolar peaks (at $125 \mathrm{~nm}$ ). The ssp-SFG spectrum at $220 \mathrm{~nm}$ becomes similar to that at $2.5 \mathrm{~nm}$ and changes to a spectrum with completely opposite peak directions at $270 \mathrm{~nm}$ [Fig. 6(a)]. Peak direction changes are also observed in the calculated $s s p$-SFG spectra at thicknesses of 410 and $560 \mathrm{~nm}$ [Fig. 6(a)].

\section{B. sps-polarization}

Figure 7 shows the calculated thickness dependence of (a) spectral shapes and (b) peak intensities for the asymmetric $\left(\mathrm{r}^{-}\right) \mathrm{C}-\mathrm{H}$ stretching mode at $2963 \mathrm{~cm}^{-1}$ and for the base line signal at $3200 \mathrm{~cm}^{-1}$ for the model system in the sps-polarization combination. The thickness dependent in- 
tensity of the SFG signal from the $\mathrm{r}^{+}$mode is quite weak and similar to the intensity of the base line signal. Since the peak intensity in the sps-polarization is very weak at thicknesses of less than $250 \mathrm{~nm}$, the intensities shown in Fig. 7(b) are magnified 50 times for clarity. Only the asymmetric $\mathrm{C}-\mathrm{H}$ stretching mode can be observed in the calculated sps-polarized SFG spectra [Fig. 7(a)]. SFG signals for the symmetric $\mathrm{C}-\mathrm{H}$ mode and the nonresonant background are very weak and neglected at all thicknesses in comparison with that of the asymmetric mode. This is reasonable since the symmetric $\mathrm{C}-\mathrm{H}$ stretching mode of the $\mathrm{CH}_{3}$ group in a well-organized monolayer or multilayer should have a very weak dipole component in the $s$-polarized IR direction and only the asymmetric $\mathrm{C}-\mathrm{H}$ stretching mode has a dipole component parallel to the surface which may interact with an $s$-polarized IR beam. As shown in Fig. 7(a), the sps-polarized SFG spectrum for a monolayer $(d \sim 2.5 \mathrm{~nm})$ shows a very small S-shaped bipolar peak. The intensity of the SF signal is roughly 20 times weaker than that for the $s s p$-polarization signal at the same thickness [Fig. 6(a)] and is too weak to be detected by the charge-coupled device (CCD) or photomultiplier (PMT) detectors used in the normal detection system of SFG spectrometers, even with long integration times. Consequently no sps-polarized SFG spectra from organic monolayers on gold substrates have yet been reported in the literature due to these low signal levels. Fundamentally this can be attributed to the small $L$ factors under sps-polarization due to the weak $s$-polarized IR electric field on the gold substrate surface [Fig. 4(b)]. The spectral shapes of the sps-SFG spectra also change significantly with thickness. As shown in Fig. 7(a), as the film thickness changes by just $20 \mathrm{~nm}$ from $120 \mathrm{~nm}$ to $140 \mathrm{~nm}$, the SFG spectral shape changes from an S-type bipolar peak $(120 \mathrm{~nm})$ to a Z-type bipolar peak $(140 \mathrm{~nm})$ with comparable intensities. The sps-SFG intensity increases rapidly when the thickness is larger than $250 \mathrm{~nm}$ and reaches an intensity maximum at $370 \mathrm{~nm}$ when it is approximately 5000 times larger than that of a monolayer [Fig. 7(b)]. It should be noted that SFG signals with such high intensity are comparable to the $s s p$-polarization signal at $220 \mathrm{~nm}$ [Fig. 3(b)] and expected to be easily detected by the standard CCD or PMT detection systems. As shown in Fig. 7(a), the sps-SFG spectrum at $2963 \mathrm{~cm}^{-1}$ shows a single intense peak at $370 \mathrm{~nm}$ after which its intensity decreases rapidly to a very small value at around $500 \mathrm{~nm}$ and changes to a weak Z-type bipolar peak shape. Subsequently the spectral intensity increases again to reach a second intensity maximum at $650 \mathrm{~nm}$ which is almost ten times stronger than that of the first maximum at $370 \mathrm{~nm}$ (results not shown in the figure).

\section{C. ppp-polarization.}

In contrast to the $s s p$ - and $s p s$-spectra, the $p p p$-spectra (Fig. 8) of the model system on the gold surface show stronger spectral intensities. The SFG signals at the gold surface modified by an $\sim 2.5 \mathrm{~nm}$ thick monolayer show an intensity maximum which is about two or three orders of magnitude stronger than that obtained for $s s p$ - and sps-polarized spectra for the same monolayer system (Figs. 6 and 7). This can be (a)

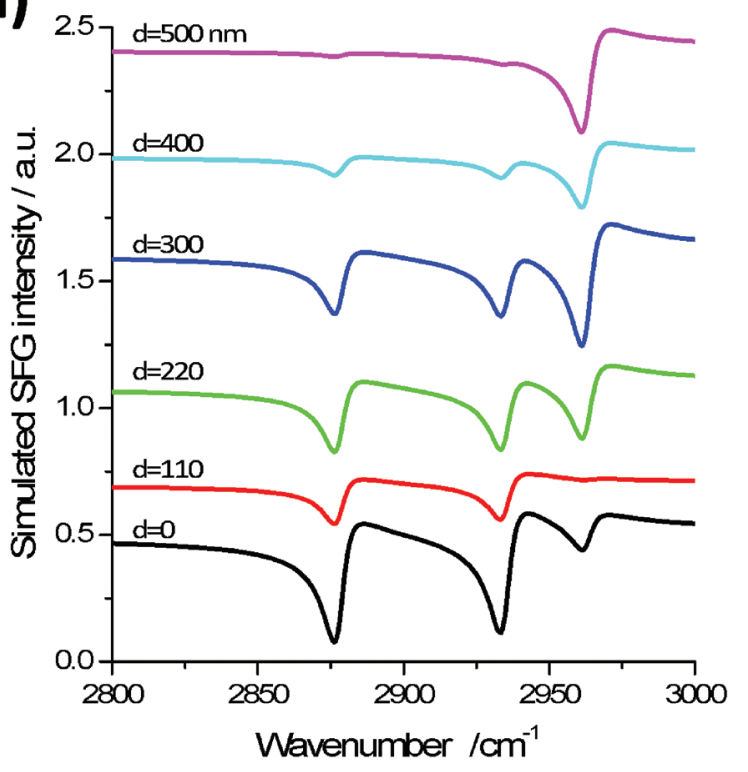

(b)

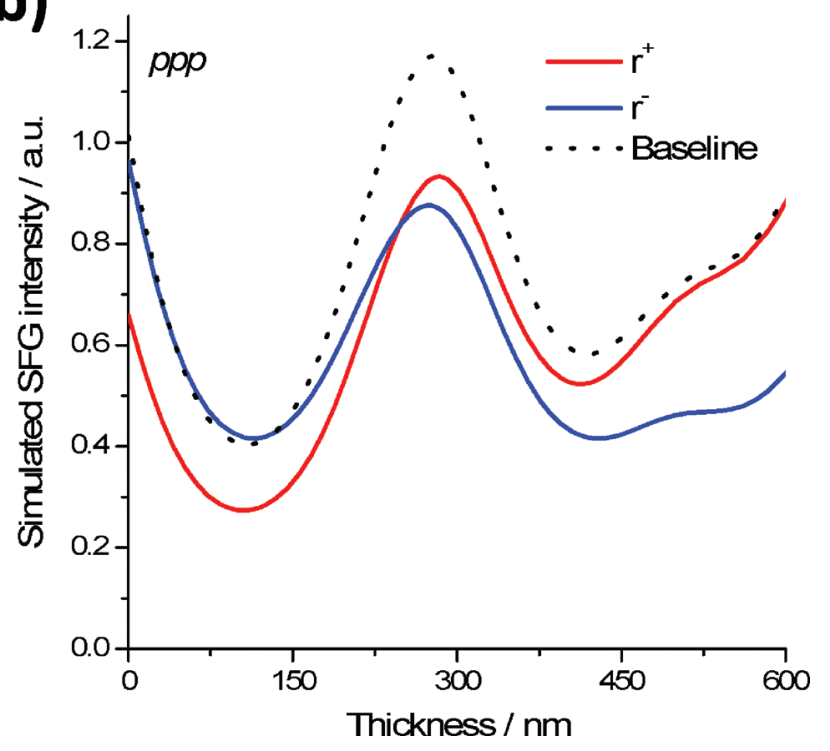

FIG. 8. Simulated results for the thickness dependence of (a) SFG spectral shapes and (b) SFG intensities for the symmetric $\left(\mathrm{r}^{+}, 2878 \mathrm{~cm}^{-1}\right)$ and asymmetric $\left(\mathrm{r}^{-}, 2963 \mathrm{~cm}^{-1}\right) \quad \mathrm{C}-\mathrm{H}$ stretching modes, and the baseline $\left(3200 \mathrm{~cm}^{-1}\right)$ for the model system in the ppp-polarization combination. Base line levels for each spectrum in (a) are displaced for clarity. See text for simulation parameters.

attributed to the higher nonresonant SFG signals from the buried film/gold interface in the ppp-polarization [Fig. 4(c)]. It is known that the $p p p-\mathrm{SFG}$ spectra for a SAM or LB monolayer on the gold substrate surface are relatively easy to obtain with a reasonable signal-to-noise ratio, especially when a Ti-sapphire laser at $800 \mathrm{~nm}$ is used as the visible beam. ${ }^{6,18,19}$ In comparison with the $s s p$ - and sps-spectra, the ppp-SFG spectra show a less pronounced thickness dependence [Fig. 8(a)]. All ppp-polarized SFG spectra show dips due to the interference with the nonresonant background from the gold substrate. The dip for the asymmetric mode becomes very weak at $110 \mathrm{~nm}$ but increases again with increasing thickness while the symmetric modes become very weak at $500 \mathrm{~nm}$. In contrast to the results for the $s s p$ - and 
(a)

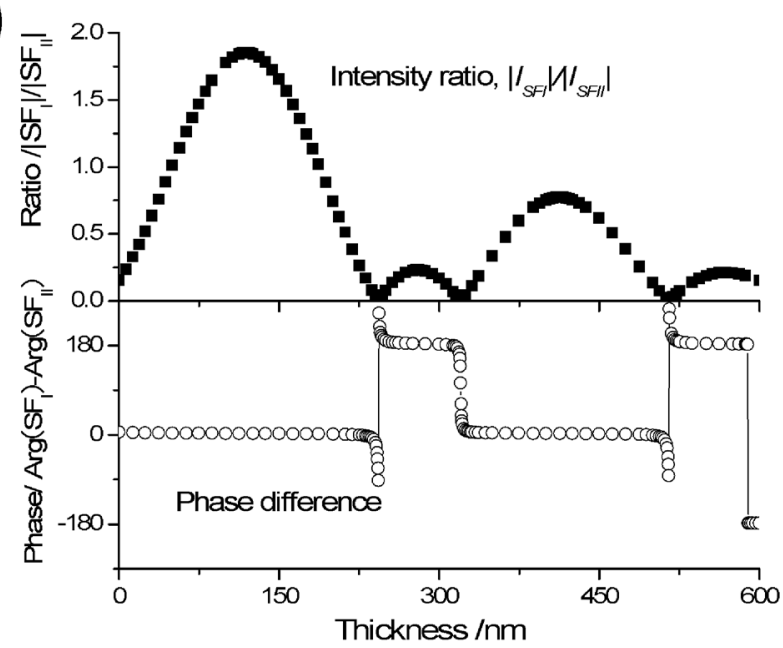

(b)

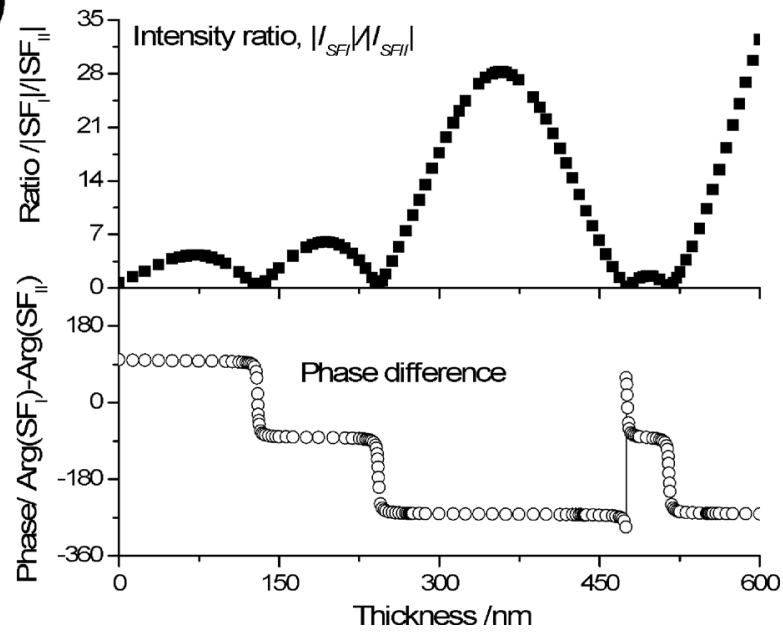

FIG. 9. Intensity ratio and phase difference for the SFG signal from the first and second interfaces of the symmetric $\left(2878 \mathrm{~cm}^{-1}\right)$ and asymmetric (2963 $\mathrm{cm}^{-1}$ ) C-H stretching modes for (a) ssp- and (b) sps-polarizations. The intensity ratio was obtained by dividing the modulus of the first term of Eq. (12a) or Eq. (12b) by the second term. The phase difference was obtained by subtracting the argument of the first term of Eq. (12a) or Eq. (12b) from that of the second term.

sps-polarizations, the SFG signals for the symmetric and asymmetric modes and for the nonresonant SFG background in the $p p p$-polarization show quite similar thickness dependences [Fig. 8(b)].

\section{Prediction of the SFG spectra}

The final SFG signal is the convolution of the SFG signals from the air/film and the film/substrate interface. The spectral features depend significantly on the relative intensities and phase differences in these two contributions. In order to understand the spectral line shapes of Figs. 6 and 7 as a function of film thickness, the intensity ratio between the resonant and nonresonant signals as well as their phase difference $^{56}$ under (a) $s s p$ - and (b) $s p s$-polarization combinations were calculated as a function of film thickness between 0 and $600 \mathrm{~nm}$ (Fig. 9). If the objective is to predict the spectral shape, then both the intensities and the phases need to be considered.
Generally, when the intensity ratio of the resonant to nonresonant components is comparable to or less than unity, the spectral shape is sensitive to the phase. For example, a peak is expected to change to a dip if the phase changes by $180^{\circ}$. However, if the intensity ratio is much larger than unity, the final SFG output is less sensitive to the phase. For example, in the ssp-polarization case [Fig. 9(a)], the phase changes by $180^{\circ}$ at thicknesses of 240,320 , and $515 \mathrm{~nm}$, where the intensity ratios are zero. Consequently the spectra will change to their mirror symmetric shapes as the film thickness crosses these thickness values. These predictions have been confirmed by the simulation results shown in Fig. 6(a). On the other hand, as the film thickness increases from 0 to $130 \mathrm{~nm}$, while the phase difference remains constant, the intensity ratio increases through unity, causing the spectra to change from dips to bipolar peaks. Similar results are also obtained for the sps-polarization [Fig. 9(b)]: The calculation predicates that the peak shape will change to its mirror symmetric shape at film thicknesses of 130, 245, 475, and 515 $\mathrm{nm}$, where the intensity ratios are zero while the spectra will be independent of the phase at a thickness of $370 \mathrm{~nm}$ and thereby show a single peak due to the intensity ratio being much greater than unity. These predictions are in good agreement with the results of Fig. 6(a). The above analysis, shown schematically in Fig. 9, can be usefully employed to quantitatively predict and analyze the actual film thickness dependent changes appearing in ssp- and sps spectra.

The prediction of the $p p p$-polarized SFG spectra is more complicated as there are four terms for both the resonant and the nonresonant signals in Eq. (12c). However, the four nonresonant terms can be simplified to one term, as the $z z z$ term is overwhelmingly greater than the others. However, there are still five terms left in Eq. (12c). Consequently there is no simple way to predict the spectral line shapes via the phase and the intensity ratio of the signals from the two interfaces for this polarization combination. However one method that can be used to predict the $p p p$-polarized SFG spectra, but only very approximately, is by separately considering the relative intensities of each resonant term's (i.e., $x x z, z x z, z x x$, or $z z z)$ interference with the dominant nonresonant term $(z z z)$, based on the same method used above for the ssp-and sps-polarization combinations (containing one resonant term only).

For example, Fig. 10 shows simulation results for the SFG spectra for the four resonant components, as well as the final SFG output, at film thicknesses of (a) $0 \mathrm{~nm}$ and (b) $110 \mathrm{~nm}$ on a gold substrate surface. The simulation results demonstrate that the contributions of the different susceptibility tensors to the final SFG spectral profile are also strongly dependent on the film thickness. For example, $\chi_{z z z}^{(2)}$ plays a dominant role for the monolayer system [Fig. 10(a)]. However, as the film thickness increases to $110 \mathrm{~nm}$, the $\chi_{x x z}^{(2)}$ component becomes the major contributor to the SFG spectrum [Fig. 10(b)]. In order to understand the spectral line shapes of $p p p$-polarized SFG spectra, the properties of each resonant component in the $p p p$-polarization combination has to be clearly addressed. 
(a)

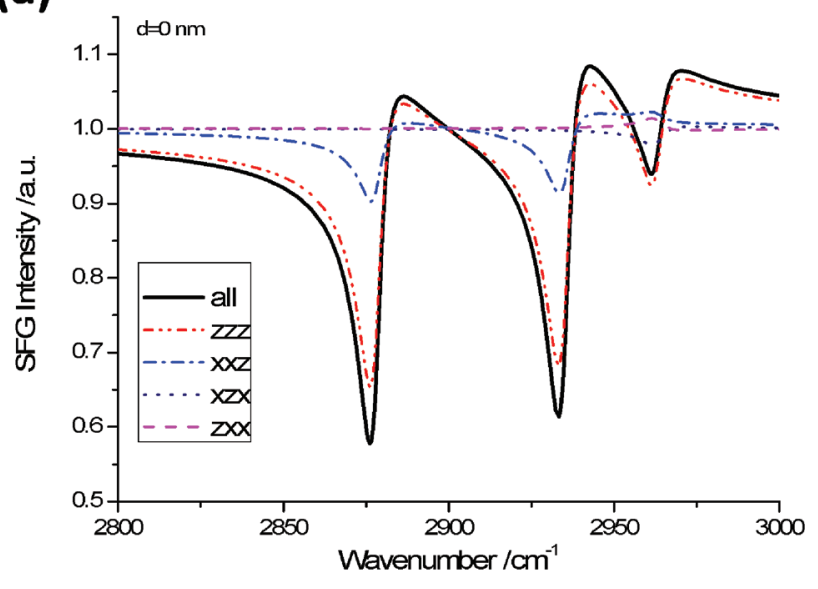

(b)

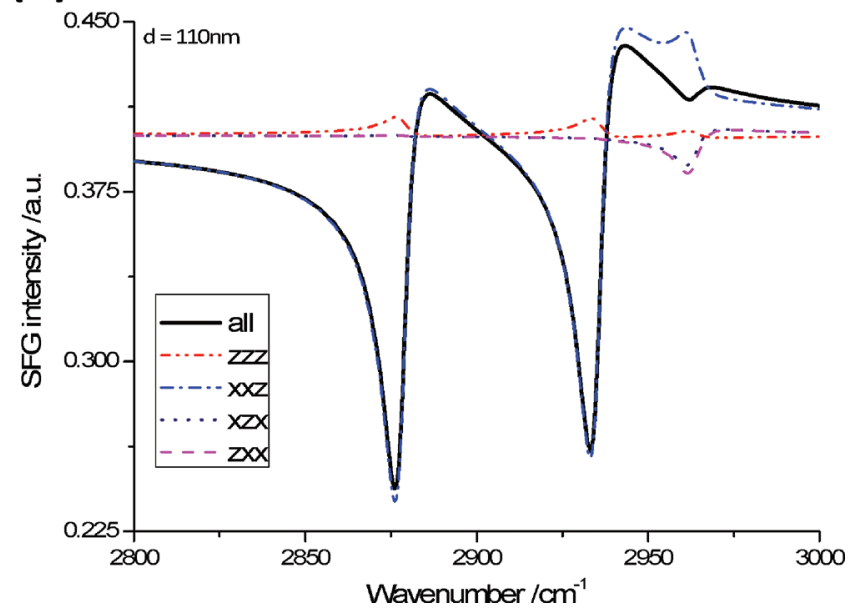

FIG. 10. Contribution of each susceptibility component, $\chi_{i j k}$, to the total SFG spectra in the ppp-polarization combination for the model system at (a) $d=0 \mathrm{~nm}$ and (b) $d=110 \mathrm{~nm}$

\section{E. Extension to other model systems}

The simulation results presented in Secs. IV A-IV C are for a specified model thin-film system, specifically one which is relevant to the authors' experimental system. However, in the experimental arrangements used by other workers, the thin film itself as well as the laser spectrometers used may be considerably different. It is thereby instructive to mention in outline the effect of these changes on the simulation results. The substantial extension of the present model system to other systems including the influence of different experimental parameters, such as the incident beam geometry (copropagating and counterpropagating), substrate (metal and dielectric materials), and thin-film structures, on the SFG spectra are discussed in the following paper. ${ }^{46}$

In the present study, the incident laser beam angles for the visible and IR beams are set at $65^{\circ}$ and $50^{\circ}$, respectively. Changing these angles will affect the $L$ factors and consequently affect the simulated spectral features at different film thicknesses. However, it was found that in order to observe noticeable changes to the interference behavior, angular variations greater than $5^{\circ}$ were required, which is in agreement with the conclusions reached by Lambert et al. ${ }^{31}$
In the present study the visible and IR beam wavelengths were chosen to be 800 and $3400 \mathrm{~nm}$ (CH stretching region). However, other visible wavelengths, such as $400 \mathrm{~nm}$ (Ref. 57) and $532 \mathrm{~nm}$ (Ref. 31) have also frequently been used in practice. Moreover, the vibrational modes of interest may lie in other frequency region. Here we briefly mention the effect of changes in the laser wavelengths. It was found that the shorter the wavelength of the incident beams, the shorter the periodicity of the multiple reflections of the beams. To summarize, a $200 \mathrm{~nm}$ change in the IR wavelength does not cause much of a change in the interference behavior, while a $50 \mathrm{~nm}$ change in the visible wavelength causes a significant change in the interference features.

As the multiple reflections take place within the thin film, it was found that the interference behavior is sensitive to the frequency dependent refractive indices of the thin film. The higher the refractive indices, the shorter the periodicities of the multiple reflections of the beams in the film, and consequently the more significant the interference appears.

The effect of three parameters relating to the surface resonant active layer, $k, R$, and $n_{m}$ in Table I, on the simulation results has been reported elsewhere. ${ }^{31}$ Generally, they only affect the relative intensities of the simulation results but not the periodicity of the multiple reflections of the beams.

\section{CONCLUSION}

In the present paper, a general model calculation is described which predicts the interference effects which may appear in the SFG spectra from a thin-film system. As a specific example, the modeling calculations were carried out for a three-layer thin-film system comprising a fatty acid monolayer/dielectric thin-film/gold substrate. The modeling calculation takes account of both the multiple reflections of each beam within the thin film and the relative phase differences at the point of coherent addition of the SFG signals from each interface. It was found that the SFG spectra varied significantly with the film thickness in both spectral intensity and shape. A general method is provided to predict the changes in the spectral shape of the SFG spectra with film thickness for the selected thin-layer system. It should be emphasized that the present calculation is restricted to a single thin film between two infinite bulk media and comprising two interfaces. More complex thin-film systems containing several interfaces ${ }^{16}$ and thin films with bulk SFG contributions are not considered in the present calculation. However, the SFG spectra from these systems can be readily predicted by extending the present modeling calculation. The model calculation also provides a robust method for unambiguously revealing quantitative structural information on the molecules at the interfaces from the observed SFG spectra. Furthermore, the method can be used to determine the film thickness by comparing the simulated and experimental spectra for a specific thin-film system provided the relevant optical and geometrical parameters are known. 


\section{ACKNOWLEDGMENTS}

This work was supported by PRESTO, Japan Science and Technology Agency (JST), and a Grant-in-Aid for Exploratory Research No. 21655074 from MEXT. We would like to thank Dr. Eric Tyrode for many valuable discussions during the work.

${ }^{1}$ Y. R. Shen and V. Ostroverkhov, Chem. Rev. (Washington, D.C.) 106, 1140 (2006)

${ }^{2}$ G. L. Richmond, Chem. Rev. (Washington, D.C.) 102, 2693 (2002).

${ }^{3}$ K. B. Eisenthal, Chem. Rev. (Washington, D.C.) 96, 1343 (1996).

${ }^{4}$ C. D. Bain, J. Chem. Soc., Faraday Trans. 91, 1281 (1995).

${ }^{5}$ A. L. Harris, C. E. D. Chidsey, N. J. Levinos, and D. N. Loiacono, Chem. Phys. Lett. 141, 350 (1987).

${ }^{6}$ L. J. Richter, T. P. Petralli-Mallow, and J. C. Stephenson, Opt. Lett. 23, 1594 (1998).

${ }^{7}$ M. Himmelhaus, F. Eisert, M. Buck, and M. Grunze, J. Phys. Chem. B 104, 576 (2000).

${ }^{8}$ A. B. Voges, H. A. Al-Abadleh, M. J. Musorrariti, P. A. Bertin, S. T. Nguyen, and F. M. Geiger, J. Phys. Chem. B 108, 18675 (2004).

${ }^{9}$ J. Hedberg, C. Leygraft, K. Cimatu, and S. Baldelli, J. Phys. Chem. C 111, 17587 (2007).

${ }^{10}$ C. Humbert, B. Busson, J. P. Abid, C. Six, H. H. Girault, and A. Tadjeddine, Electrochim. Acta 50, 3101 (2005).

${ }^{11}$ C. Weeraman, A. K. Yatawara, A. N. Bordenyuk, and A. V. Benderskii, J. Am. Chem. Soc. 128, 14244 (2006).

${ }^{12}$ S. Ye, S. Nihonyanagi, and K. Uosaki, Phys. Chem. Chem. Phys. 3, 3463 (2001).

${ }^{13}$ Z. Chen, Y. R. Shen, and G. A. Somorjai, Annu. Rev. Phys. Chem. 53, 437 (2002).

${ }^{14}$ H. Rangwalla, A. D. Schwab, B. Yurdumakan, D. G. Yablon, M. S. Yeganeh, and A. Dhinojwala, Langmuir 20, 8625 (2004).

${ }^{15}$ S. Ye, S. Morita, G. F. Li, H. Noda, M. Tanaka, K. Uosaki, and M. Osawa, Macromolecules 36, 5694 (2003).

${ }^{16}$ P. Guyot-Sionnest, J. H. Hunt, and Y. R. Shen, Phys. Rev. Lett. 59, 1597 (1987).

${ }^{17}$ G. Ma and H. C. Allen, Langmuir 23, 589 (2007).

${ }^{18}$ S. Ye, H. Noda, S. Morita, K. Uosaki, and M. Osawa, Langmuir 19, 2238 (2003)

${ }^{19}$ S. Ye, H. Noda, T. Nishida, S. Morita, and M. Osawa, Langmuir 20, 357 (2004)

${ }^{20}$ T. Nishida, C. M. Johnson, J. Holman, M. Osawa, P. B. Davies, and S. Ye, Phys. Rev. Lett. 96, 077402 (2006).

${ }^{21}$ R. N. Ward, P. B. Davies, and C. D. Bain, J. Phys. Chem. 97, 7141 (1993).

${ }^{22}$ J. Holman, P. B. Davies, T. Nishida, S. Ye, and D. J. Neivandt, J. Phys. Chem. B 109, 18723 (2005).

${ }^{23}$ V. Ostroverkhov, G. A. Waychunas, and Y. R. Shen, Phys. Rev. Lett. 94, 046102 (2005).

${ }^{24}$ S. Nihonyanagi, S. Yamaguchi, and T. Tahara, J. Chem. Phys. 130, 204704 (2009).

${ }^{25}$ M. G. Brown, E. A. Raymond, H. C. Allen, L. F. Scatena, and G. L. Richmond, J. Phys. Chem. A 104, 10220 (2000).

${ }^{26}$ W. Gan, Z. Zhang, R. R. Feng, and H. F. Wang, J. Phys. Chem. C 111, 8726 (2007)
${ }^{27}$ T. Ishiyama and A. Morita, J. Phys. Chem. C 113, 16299 (2009).

${ }^{28}$ M. A. Belkin, T. A. Kulakov, K. H. Ernst, L. Yan, and Y. R. Shen, Phys. Rev. Lett. 85, 4474 (2000).

${ }^{29}$ J. Wang, M. L. Clarke, and Z. Chen, Anal. Chem. 76, 2159 (2004).

${ }^{30}$ D. Wilk, D. Johannsmann, C. Stanners, and Y. R. Shen, Phys. Rev. B 51, 10057 (1995).

${ }^{31}$ A. G. Lambert, D. J. Neivandt, A. M. Briggs, E. W. Usadi, and P. B. Davies, J. Phys. Chem. B 106, 5461 (2002).

${ }^{32}$ C. Hirose, H. Ishida, K. Iwatsu, N. Watanabe, J. Kubota, A. Wada, and K. Domen, J. Chem. Phys. 108, 5948 (1998).

${ }^{33}$ M. L. Clarke, Ph.D. thesis, The University of Michigan, 2006.

${ }^{34}$ Y. R. Shen, The Principles of Nonlinear Optics (Wiley, New York, 1984).

${ }^{35}$ E. Hecht, Optics, 4th ed. (Pearson Education, San Francisco, 2002).

${ }^{36} \mathrm{P}$. Yeh, Optical Waves in Layered Media (Wiley, New York, 1988).

${ }^{37}$ J. E. Sipe, J. Opt. Soc. Am. B 4, 481 (1987).

${ }^{38}$ D. S. Bethune, J. Opt. Soc. Am. B 8, 367 (1991).

${ }^{39}$ M. B. Feller, W. Chen, and Y. R. Shen, Phys. Rev. A 43, 6778 (1991).

${ }^{40}$ P. T. Wilson, K. A. Briggman, W. E. Wallace, J. C. Stephenson, and L. J. Richter, Appl. Phys. Lett. 80, 3084 (2002).

${ }^{41}$ K. A. Briggman, J. C. Stephenson, W. E. Wallace, and L. J. Richter, J. Phys. Chem. B 105, 2785 (2001).

${ }^{42}$ It should be mentioned that the formic acid thin layers formed in the work do not have centrosymmetric structure, hence, give a large contribution to the SFG signal from the bulk film, which is different from the thin-layer model system discussed in the present study.

${ }^{43}$ A. G. Lambert, D. J. Neivandt, A. M. Briggs, E. W. Usadi, and P. B. Davies, J. Phys. Chem. B 106, 10693 (2002).

${ }^{44}$ J. Holman, P. B. Davies, and D. J. Neivandt, J. Phys. Chem. B 108, 1396 (2004).

${ }^{45}$ S. J. McGall, P. B. Davies, and D. J. Neivandt, J. Phys. Chem. B 108, 16030 (2004).

${ }^{46}$ Y. Tong, Y. Zhao, N. Li, Y. Ma, M. Osawa, P. B. Davies, and S. Ye, J. Chem. Phys. 133, 034705 (2010).

${ }^{47}$ C. Hirose, N. Akamatsu, and K. Domen, Appl. Spectrosc. 46, 1051 (1992).

${ }^{48}$ A. J. Moad and G. J. Simpson, J. Phys. Chem. B 108, 3548 (2004).

${ }^{49}$ C. Hirose, H. Yamamoto, N. Akamatsu, and K. Domen, J. Phys. Chem. 97, 10064 (1993)

${ }^{50}$ X. Wei, S. C. Hong, X. W. Zhuang, T. Goto, and Y. R. Shen, Phys. Rev. E 62, 5160 (2000).

${ }^{51}$ X. Zhuang, P. B. Miranda, D. Kim, and Y. R. Shen, Phys. Rev. B 59, 12632 (1999).

${ }^{52}$ As described in Eq. (12), the ppp-polarized SFG signal contains four terms, namely, $x z x, x x z, z x x$, and $z z z$. However, in the case of the present thin-film system, the $L$ factors of the buried interface for the $x z x, x x z$, and $z x x$ terms in Eq. (12) are three orders of magnitude weaker than the $z z z$ term (results not shown) and can therefore be omitted. Consequently, the ppp-polarized SFG signal from the bottom interface is represented by the $z z z$ component alone.

${ }^{53}$ C. Hirose, N. Akamatsu, and K. Domen, J. Chem. Phys. 96, 997 (1992).

${ }^{54}$ H. F. Wang, W. Gan, R. Lu, Y. Rao, and B. H. Wu, Int. Rev. Phys. Chem. 24, 191 (2005).

${ }^{55}$ M. J. Weber, Handbook of Optical Materials (CRC, Boca Raton, 2002).

${ }^{56}$ The definition of the phase difference here is slightly different from that reported by Lambert et al.: Here the phase difference arising from the multiple reflections of each beam was also included.

${ }^{57}$ T. A. Ishibashi and H. Onishi, Appl. Spectrosc. 56, 1298 (2002). 